\title{
THE APPROXIMATE FUNCTIONAL FORMULA FOR THE THETA FUNCTION AND DIOPHANTINE GAUSS SUMS
}

\author{
E. A. COUTSIAS AND N. D. KAZARINOFF
}

\begin{abstract}
We consider the polygonal lines in the complex plane $\mathbb{C}$ whose $N$ th vertex is defined by $S_{N}=\sum_{n=0}^{N^{\prime}} \exp \left(i \omega \pi n^{2}\right)$ (with $\omega \in \mathbb{R}$ ), where the prime means that the first and last terms in the sum are halved. By introducing the discrete curvature of the polygonal line, and by exploiting the similarity of segments of the line, for small $\omega$, to Cornu spirals (C-spirals), we prove the precise renormalization formula
\end{abstract}

$$
\begin{aligned}
& \left|\sum_{k=0}^{N}{ }^{\prime} \exp \left(i \omega \pi k^{2}\right)-\frac{\exp (\operatorname{sgn}(\omega) i \pi / 4)}{\sqrt{|\omega|}} \sum_{k=0}^{n} ' \exp \left(-i \frac{\pi}{\omega} k^{2}\right)\right| \\
& \quad \leq C\left|\frac{\omega N-n}{\omega}\right|, \quad 0<|\omega|<1,
\end{aligned}
$$

where $N=[[n / \omega]]$, the nearest integer to $n / \omega$ and $1<C<3.14$.

This formula, which sharpens Hardy and Littlewood's approximate functional formula for the theta function, generalizes to irrationals, as a Diophantine inequality, the well-known sum formula of Gauss. The geometrical meaning of the relation between the two limits is that the first sum is taken to a point of inflection of the corresponding $\mathrm{C}$-spirals. The second sum replaces whole $\mathrm{C}$-spirals of the first by unit vectors times scale and phase factors. The block renormalization procedure implied by this replacement is governed by the circle map

$$
\left.\omega \rightarrow-\frac{1}{\omega} \quad(\bmod 2), \omega \in\right]-1,+1[\backslash\{0\},
$$

whose orbits are analyzed by expressing $\omega$ as an even continued fraction.

\section{INTRODUCTION}

The quadratic Gauss sum formula

$$
\sum_{0}^{q} e^{2 \pi i k^{2} / q}=\sqrt{\frac{q}{2}} e^{i \pi / 4}\left(1+e^{-i \pi q / 2}\right)
$$

can be easily generalized to the Cauchy-Kronecker formula [1], [5]

$$
\sum_{0}^{q} ' e^{i \pi\left(\frac{p}{q}\right) k^{2}}=e^{i \pi / 4} \sqrt{\frac{q}{p}} \sum_{0}^{p}{ }^{\prime} e^{-i \pi\left(\frac{q}{p}\right) k^{2}}, \quad p q \equiv 0 \quad(\bmod 2) .
$$

Here a primed sum indicates that the first and last terms are halved. Gauss used (3) for one of his shorter proofs of the law of quadratic reciprocity ([1], p. 200).

Received by the editors January 25, 1995 and, in revised form, December 4, 1995.

1991 Mathematics Subject Classification. Primary 11G10; Secondary 11L05, 11L07, 11J25, 11J70.

(C) 1998 American Mathematical Society 
Formula (4) is related to the Jacobi imaginary transformation giving a functional equation satisfied by the theta function $\vartheta_{3}(v, \tau)$. As is well known, for

$$
\vartheta_{3}(v, \tau):=\sum_{-\infty}^{\infty} e^{i \pi \tau k^{2}+2 \pi i k v} \quad(\Im(\tau)>0)
$$

we have [5], for $v=0$,

$$
\vartheta_{3}(0, \tau)=(i / \tau)^{1 / 2} \vartheta_{3}(0,-1 / \tau),
$$

or

$$
\sum_{-\infty}^{\infty} e^{i \pi \tau k^{2}}=(i / \tau)^{1 / 2} \sum_{-\infty}^{\infty} e^{-i \pi k^{2} / \tau} .
$$

This formula is useful, e.g. for estimating $\vartheta_{3}$ for small $\Im \tau$. Obviously, (5) diverges if $\Im \tau \rightarrow 0$. A limit process, setting $z=p / q+i \epsilon$ in (7) with $\epsilon>0$ and letting $\epsilon \rightarrow 0^{+}$, can be used to establish the validity of (4) [5]. For $z=\omega+i \epsilon, \omega \in \mathbb{R} \backslash Q$, however, the problem of determining the limiting behavior of $\vartheta_{3}$ near the real axis is considerably more difficult. Setting $\omega=\Re \tau$, one has instead the approximate formula ([11], Thm. 2.128, p. 209, given here in a slightly sharper form, due to J.R. Wilton [23]), adapted to our case:

$$
\begin{gathered}
\sum_{0}^{N}{ }^{\prime} e^{i \pi \omega k^{2}}=(i / \omega)^{1 / 2} \sum_{0}^{[N \omega]}{ }^{-i \pi k^{2} / \omega}+R, \\
|R|<2.426+1.577|\omega|^{-\frac{1}{2}} .
\end{gathered}
$$

Hardy and Littlewood stated and proved a slightly less precise form of this result, which became known as "the approximate functional formula for the theta function". For their original proof they used a rather lengthy contour integral method, akin to a method introduced by Kronecker [15] for his proof of (3). Their estimate was improved by Mordell [21] using a simpler variant of the contour integral method. Wilton's proof of the sharp form (8) uses the Poisson sum formula. This latter proof is the basis for our Diophantine estimates (14), derived in Section 2. Formula (8) was extended by van der Corput [6] to a wide range of sums of the type $\sum g(k) e^{2 \pi i f(k)}$. Sums of this type arise, e.g., in the analysis of the distribution of fractional parts of functions [22], [9], and can be useful in deriving estimates for the number of integral lattice points in multidimensional domains [7], [13].

In [11], Hardy and Littlewood related the properties of the sums (8) to the Euclidean continued fraction expansion of $\omega$. As we showed in [8], however, the continued fraction expansion of $\omega$ in even integers gives a direct interpretation, and in Sec. 3 we study some of the properties of such expansions. Berry and Goldberg [2] gave an analysis of the renormalization using Euclidean continued fractions. Their work was overviewed in [20], where even continued fractions were also mentioned but not analyzed.

Hardy and Littlewood's estimate was derived by them as part of their work on studying the convergence properties of Fourier series. Their asymptotic analysis applies for $N \rightarrow \infty, \omega$ kept fixed. This somewhat masks the renormalization and selfsimilarity properties of sums of this type which are the motivation of our work. Indeed, formula (8), viewed as an approximate identity, applicable for arbitrary (but fixed) $N$, has the disadvantage that the error term diverges as $\omega \rightarrow 0$. Thus, this form of the formula, although suggestive, does not elucidate the detailed properties 


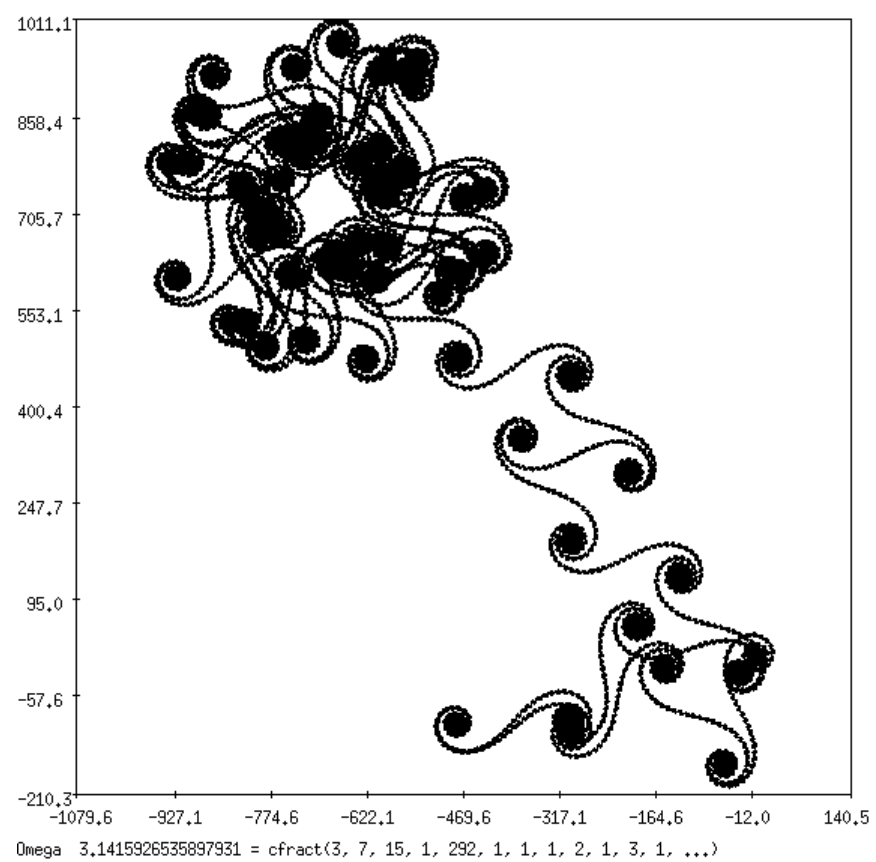

(a)

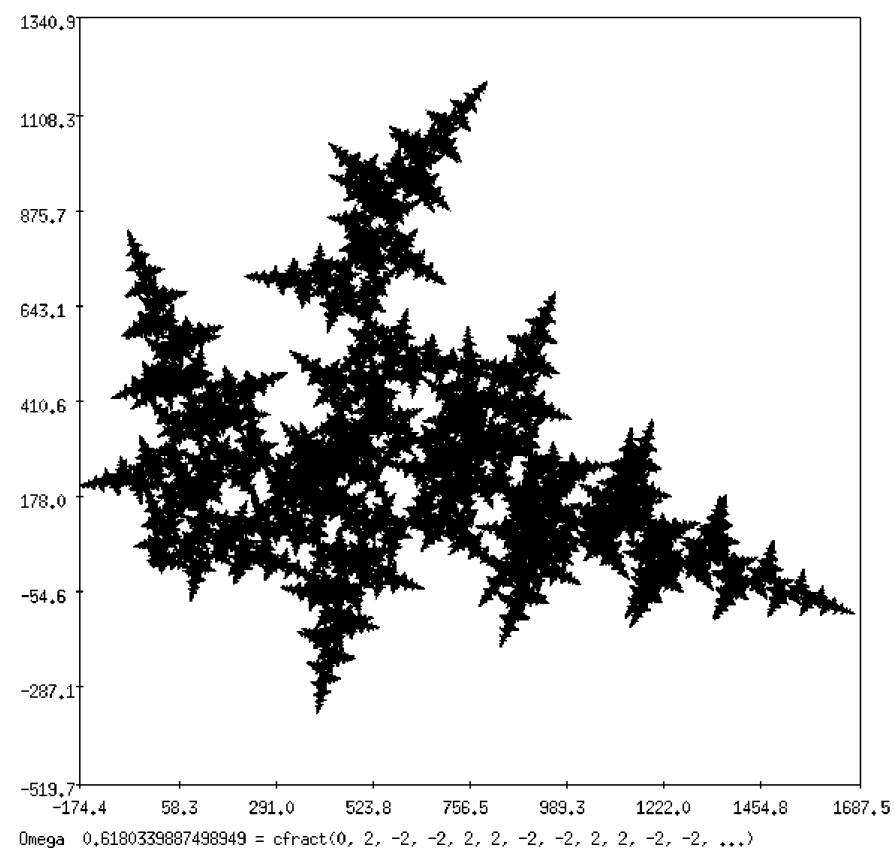

(b)

Figure 1. The partial sums for $\pi$ (top) and the golden ratio (bottom); in both cases $N=2 \times 10^{6}$ 
of the pattern, such as its self-similarity and scalings, quite satisfactorily, and the deductions drawn from it must rely as much on this formula as on computations for their substantiation. In order to deduce the self-similarity of the pattern for finite values of $n$ as well as for small $\omega$, one needs a more detailed argument, and a careful discussion of the appropriate limits in the sums. Our renormalization argument, given heuristically in [8], shows that the expression in (8) can be thought of as the result of summing the left hand side in blocks and should be increasingly accurate as $\omega \rightarrow 0$.

The key idea, given in [8], is that the partial sums of (8) form Cornu-like spirals in the complex plane $(\mathbb{C})$; see Figure 1a, in which the first 44,000 points in the sequence $\left\{S_{n}\right\}$ are plotted for $\omega=\pi$, and Figure 1b, in which the first 30,000 points in the sequence $\left\{S_{n}\right\}$ are plotted for $\omega=(\sqrt{5}-1) / 2$, the Golden Mean. If we define the discrete radius of curvature $R_{N}$ of the pattern at a point $S_{N}$ as the radius of the circle defined by the point and its successor and predecessor in the sequence, it is straightforward to show that it has maxima (inflection points of the pattern) at the points $S_{N}$, where

$$
S_{N}=\sum_{k=0}^{N} e^{i \pi \omega k^{2}}
$$

for $N=n_{2 \ell}$, and minima (cusps of the pattern) at the points $S_{N}$, for $N=n_{2 \ell+1}$, with $n_{k}$ given by

$$
n_{k}=\left[\left[\frac{k}{2 \omega}\right]\right] .
$$

Here $[[\alpha]]$ denotes as usual the closest integer to $\alpha$.

This is a consequence of the following definition: The discrete curvature $K_{N}$ of the graph of $\left\{S_{N}\right\}$ at $N$ (except at cusps) is the average of the curvatures of the circles passing through the points $\left\{S_{N-2}, S_{N-1}, S_{N}\right\}$ and $\left\{S_{N-1}, S_{N}, S_{N+1}\right\}$. Thus

$$
K_{N}=\frac{1}{2}\left(\frac{1}{R_{N-1}}+\frac{1}{R_{N}}\right),
$$

with

$$
R_{N}=\frac{1}{2}\left|\csc \left(\psi_{N} / 2\right)\right|,
$$

where $\psi_{N}$ is the change in argument between the $(N+1)$-st and $N$-th term in the sum, that is,

$$
\psi_{N}=\pi \omega\left((N+1)^{2}-N^{2}\right)=\pi \omega(2 N+1) .
$$

From this it follows that minima of $K_{N}$ are found at points of inflection, where $\psi_{N}$ is closest to $2 \ell \pi$ for some integer $\ell$ (see [8], p. 301). For $\omega$ irrational, $R_{N}$ never becomes $\infty$ or 0 .

Numerical computations of the finite sum (9) indicated a haunting selfsimilarity between the patterns generated by various partial sums of this form. In [8] our heuristic discussion led to a renormalization argument. We related the vector $S_{n_{2 \ell+1}}-S_{n_{2 \ell-1}}$ which joins the two successive cusps delimiting the $l$-th spiral to the vector $z_{n_{2 \ell}}=\exp \left(i \pi \omega n_{2 \ell}^{2}\right)$ ("mid-vector" of the $l$-th spiral), observing that as $\omega \rightarrow 0$ the pattern between consecutive cusps closely resembles a Cornu spiral. Thus, the vector joining successive cusps is at approximately $\pi / 4$ radians to the mid-vector, 
to the left or right according to whether $-1 / \omega(\bmod 2)$ lies in the positive or negative half of the interval $(-1,1)$, and its length is approximately $\sqrt{|\omega|}$ in agreement to the value of the Fresnel integral between $-\infty$ and $+\infty$, scaled appropriately.

One notices that practically all of the points in the graphs of $S_{n}$ lie near to cusps. Moreover, because $\left|S_{n+1}-S_{n}\right|=\left|z_{n+1}\right|=1$, the sums $S_{n}$ inaccurately approximate the values of integrals corresponding to full spirals, although the error is reduced by halving the first and last terms. The sharp error bound claimed above is found if we consider the block sums between successive cusps together with a refinement of Wilton's [23] method which replaces his use of the triangle inequality by a detailed cancellation of terms in the remainder. This reasoning led us to the Diophantine approximate functional formula, if $n \in \mathbb{Z}^{+}$and $N=[[n / \omega]]$,

$$
\left|\sum_{0}^{N}{ }^{\prime} e^{i \pi \omega k^{2}}-\frac{e^{i \frac{\pi}{4} \operatorname{sgn}(\omega)}}{\sqrt{|\omega|}} \sum_{0}^{n}{ }^{\prime} e^{-i \frac{\pi}{\omega} k^{2}}\right| \leq C\left|\frac{\omega N-n}{\omega}\right|, \quad C<3.14 .
$$

The resolution of the apparent contradiction between our result, which becomes arbitrarily precise as $\omega \rightarrow 0$ (for $N=[[n / \omega]],|\omega|<1$, which implies, but is not equivalent to the relation $n=[[N \omega]])$ and Hardy and Littlewood's, in which the error term blows up as $\omega \rightarrow 0$, is now clear: since they are not summing over integral numbers of spirals, their error term is comparable to the size of one spiral in the renormalized sum, or $O(1 / \sqrt{|\omega|})$. Our estimate, as given by (14), is uniform in $\omega$. Hence, it results in arbitrarily precise formulas if we let $N$ (and $n$ ) approach infinity through the sequence of denominators $q_{k}$ (numerators $p_{k}$ ) of partial convergents of the irrational $\omega$. For it is in this case that $\left|\epsilon_{n}\right|=|\omega N-n|$ and $\left|\epsilon_{n} / \omega\right|=|N-n / \omega|$ are smallest and both tend to zero. In the worst case, if $|\omega|$ is small and $n / N$ is not a partial convergent of the Euclidean continued fraction for $\omega$, our error term is not large because $\left|\epsilon_{n} / \omega\right|<1 / 2$, since $N=[[n / \omega]]$. Of course, the smallest values of $\left|\epsilon_{n}\right|$ are found if $N$ is a denominator and $n$ is the corresponding numerator of a convergent of $\omega$. Examining (14), we see that a repetition of the process to further reduce the sum would not necessarily produce a small error, since $n$ is not necessarily a convergent denominator of $-1 / \omega(\bmod 2)$. Only if it is, can a repeated renormalization be performed; hence in Sec. 3 we give a careful analysis of the renormalization map $\omega \rightarrow-1 / \omega(\bmod 2)$ defined in $]-1,1[\backslash\{0\}$. Alternative discussions of the scale invariance properties of the pattern using the Euclidean continued fraction expansion of $\omega$ were given also by Loxton [17], Deshouillers [10], Callot and Diener [4] and Berry and Goldberg [2]. The latter also make the connection to renormalization, from a slightly different viewpoint than the one presented in [8]. For an analysis from the point of view of dimension theory, see [9] and [18] where a theory for the dimension of plane curves is developed. Its results are applied, among other things, to curves defined using the left hand side of (8), with interesting conclusions relating the dimension to the Diophantine properties of the irrational $\omega$. Among the interesting applications of that work we mention its connection between the properties of the graph and the theory of uniform distributions for arithmetic sequences. A qualitative discussion of the properties of the sums was given recently in [20], following the ideas of [18] and [2]. Up to date reviews of the method of exponential sums and its applications can be found in the monographs by Korobov [14] and Montgomery [19].

This article is organized as follows: In Sec. 2 and App. A we give a proof of the Diophantine error estimate. In Sec. 3 we examine the expansion of the irrational 
number $\omega$ in terms of a continued fraction with even, positive or negative, entries, and we relate its properties to the main estimate in Theorem 2.1 below.

\section{The Diophantine ERror EStimate}

Consider the generalized Gauss sum

$$
\left.S(a, b ; \omega):=\sum_{a}^{b}{ }^{\prime} e^{i \pi \omega k^{2}}, \quad|\omega| \in\right] 0,1[,
$$

where the prime means that the first and last terms in the sum are halved. Our main result is the following.

Theorem 2.1. There exists a $C$ with $1.0<C<3.14$ (whose upper bound is independent of $n$ and $\omega \in]-1,+1[\backslash\{0\})$ such that

$$
\begin{aligned}
\mid S(0, & N ; \omega)-\frac{e^{i \frac{\pi s g n(\omega)}{4}}}{\sqrt{|\omega|}} S(0, n ;-1 / \omega) \mid \\
& \leq\left|\frac{\epsilon_{n}}{\omega}\right|\left(1.00+|\omega| 1.116081+|\omega|^{2} 1.023237\right) \\
& \leq C\left|\frac{\epsilon_{n}}{\omega}\right|
\end{aligned}
$$

where $N=[[n / \omega]]$, the closest integer to $n / \omega$, and $\epsilon_{n}=N \omega-n$.

Remark 2.1. If $\omega$ is rational, then $n$ can be found so that $\epsilon_{n}=0$, and our result reduces to the classical formula (4).

Proof. It is convenient to consider

$$
S(-N, N ; \omega)=2 S(0, N ; \omega) .
$$

We apply the Poisson summation formula [12] to (15), to find

$$
S(a, b ; \omega)=P V \sum_{k=-\infty}^{\infty} \int_{a}^{b} e^{i \pi \omega t^{2}+2 \pi i k t} d t,
$$

where the infinite series must be understood in the principal value sense, $P V \sum_{-\infty}^{\infty}$ $=\lim _{K \rightarrow \infty} \sum_{-K}^{K}$.

The sum can be decomposed into the pieces which offer the significant contribution and an error term. The decomposition, which follows Wilton [23], can be understood if we let $\phi_{k}(t)=\frac{1}{2} \omega t^{2}+k t, \phi_{k}^{\prime}=\omega t+k$. With Berry and Goldberg [2] (see also Montgomery [19]), we note that the phase $\phi_{k}(t)$ of the $k$-th integrand becomes stationary at the point $t_{k}=-k / \omega$ (i.e. $\phi_{k}^{\prime}\left(t_{k}\right)=0$ ). Choosing $N$ so that it is equal to $\left[\left[\frac{n}{\omega}\right]\right]$ for some integer $n$ (and hence $n=[[N \omega]]$, the nearest integer to the irrational $N \omega$ ), we decompose the sum

$$
S(-N, N ; \omega)=\sum_{k=-\infty}^{\infty} \int_{-N}^{N} \exp \left(2 \pi i \phi_{k}(t)\right) d t
$$


according to how the stationary point $t_{k}$ of the phase of the $k$-th term is situated with respect to the interval $(-N, N)$ :

$$
\begin{aligned}
S(-N, N ; \omega) & =\left(\sum_{k=-\infty}^{-n-1}+\sum_{k=-n+1}^{n-1}+\sum_{k=n+1}^{+\infty}\right) \int_{-N}^{N} \exp \left(2 \pi i \phi_{k}(t)\right) d t \\
& +\int_{-N}^{N} \exp \left(2 \pi i \phi_{-n}\right) d t+\int_{-N}^{N} \exp \left(2 \pi i \phi_{n}\right) d t \\
& =\sigma_{1}+\sigma_{2}-\delta_{2}+\sigma_{3}-\delta_{3}-\delta_{4},
\end{aligned}
$$

where

$$
\sigma_{1}=\left(\sum_{k=-\infty}^{-n-1}+\sum_{k=n+1}^{+\infty},\right) \int_{-N}^{N} \exp \left(2 \pi i \phi_{k}\right) d t
$$

(terms with stationary phase point outside interval of integration are left unchanged),

$$
\sigma_{2}=\sum_{k=-n+1}^{n-1} \int_{-\infty}^{\infty} \exp \left(2 \pi i \phi_{k}\right) d t
$$

(terms with stationary phase point in the interval $(-N, N)$ have their domain of integration extended to infinity),

$$
\delta_{2}=\sum_{k=-n+1}^{n-1}\left(\int_{-\infty}^{-N}+\int_{N}^{\infty}\right) \exp \left(2 \pi i \phi_{k}\right) d t
$$

(compensation for extended domains of integration in $\sigma_{2}$ ),

$$
\sigma_{3}=\int_{-\infty}^{n / \omega} \exp \left(2 \pi i \phi_{-n}\right) d t+\int_{-n / \omega}^{\infty} \exp \left(2 \pi i \phi_{n}\right) d t
$$

(terms with stationary phase point at $t_{ \pm n}=\mp n / \omega$ lie within distance less than $1 / 2$ from the endpoint $\mp N$ of the domain of integration, respectively and they have their domains modified as follows: move the opposite endpoint from $\pm N$ to $\pm \infty$, and move the near endpoint from $\mp N$ to the respective stationary phase point $\mp n / \omega ; \delta_{3}, \delta_{4}$ give the corresponding compensations),

$$
\begin{gathered}
\delta_{3}=\int_{-\infty}^{-N} \exp \left(2 \pi i \phi_{-n}\right) d t+\int_{N}^{\infty} \exp \left(2 \pi i \phi_{n}\right) d t, \\
\delta_{4}=\int_{N}^{n / \omega} \exp \left(2 \pi i \phi_{-n}\right) d t+\int_{-n / w}^{-N} \exp \left(2 \pi i \phi_{n}\right) d t .
\end{gathered}
$$

We now produce, in a series of lemmas, the expressions and estimates necesary for our estimation of $C$, claimed in Theorem 2.1.

\section{Lemma 2.1.}

$$
\left|\delta_{4}\right| \leq 2\left|\frac{\omega N-n}{\omega}\right|=2|\epsilon / \omega|
$$

Proof.

$$
\delta_{4}=2 \int_{N}^{n / \omega} \exp \left(i \pi\left(\omega t^{2}-2 n t\right)\right) d t=2 e^{-i \pi n^{2} / \omega} \int_{0}^{n / \omega-N} e^{i \pi \omega s^{2}} d s .
$$


Note: this term must be compared to the term $1.577 \omega^{-1 / 2}$ in the error term of Wilton. Our estimate is smaller, since the length of our interval of integration is $|\epsilon / \omega|<1 / 2$. Wilton needs to use his larger estimate since his domain of integration can be considerably larger, involving several cycles of the integrand. Indeed, since $n=[[N \omega]]$, we have that $|n / \omega-N| \leq 1 /(2 \omega)$, which can be quite large.

\section{Lemma 2.2.}

$$
\sigma_{2}+\sigma_{3}=\frac{e^{i \pi / 4}}{\sqrt{\omega}} \sum_{k=-n}^{n} e^{-i \pi k^{2} / \omega}
$$

Proof. We have

$$
\int_{-\infty}^{\infty} e^{2 \pi i \phi_{k}} d t=e^{-\pi i k^{2} / \omega} \int_{-\infty}^{\infty} e^{i \pi \omega s^{2}} d s=\frac{e^{i \pi / 4}}{\sqrt{\omega}} e^{-i \pi k^{2} / \omega}
$$

and

$$
\begin{aligned}
\int_{-n / \omega}^{\infty} e^{2 \pi i \phi_{n}} d t & =\int_{-n / \omega}^{\infty} e^{i \pi \omega\left(t^{2}+\frac{2 n}{\omega} t+\frac{n^{2}}{\omega^{2}}\right)} e^{-i \pi n^{2} / \omega} d t \\
& =e^{-i \pi n^{2} / \omega} \int_{0}^{\infty} e^{i \pi \omega s^{2}} d s=\frac{1}{2} \frac{e^{i \pi / 4}}{\sqrt{\omega}} e^{-i \pi n^{2} / \omega}
\end{aligned}
$$

and the desired expression follows.

Our task now is to estimate the error term $\sigma_{1}-\delta_{2}-\delta_{3}$. We will show that it is bounded by a quantity similar to the bound found for $\delta_{4}$. We have

Lemma 2.3.

$$
\sigma_{1}-\delta_{2}-\delta_{3}=\left.2 \frac{e^{\pi i \omega N^{2}}}{2 \pi i} \sum_{\ell=0}^{m} \frac{\omega^{\ell}}{(2 \pi i)^{\ell}(2 \ell) ! !} \frac{d^{2 \ell}}{d s^{2 \ell}} \psi(s)\right|_{s=\epsilon}+I,
$$

where $\psi(s)=\pi \cot \pi s-\frac{1}{s}$ is regular for $|s|<1$ and

$$
\begin{aligned}
I=\frac{(2 m+1) ! ! \omega^{m+1}}{(2 \pi i)^{m+1}}\{ & \left(\sum_{k=-\infty}^{-n-1}+\sum_{k=n+1}^{\infty}\right) \int_{-N}^{N} \\
& \left.-\sum_{k=-n}^{n-1} \int_{-\infty}^{-N}-\sum_{k=-n+1}^{n} \int_{N}^{\infty}\right\} \frac{e^{\pi i\left(\omega t^{2}+2 k t\right)}}{(\omega t+k)^{2 m+2}} d t .
\end{aligned}
$$

Proof. Integrating by parts $m+1$ times, one finds that

$$
\begin{aligned}
\int_{x}^{y} e^{i \pi\left(\omega t^{2}+2 k t\right)} d t & =\left.e^{\pi i\left(\omega t^{2}+2 k t\right)}\left\{\frac{1}{2 \pi i(\omega t+k)}+\sum_{\ell=1}^{m} \frac{(2 \ell-1) ! ! \omega^{\ell}}{(2 \pi i)^{\ell+1}(\omega t+k)^{2 \ell+1}}\right\}\right|_{x} ^{y} \\
& +\frac{(2 m+1) ! ! \omega^{m+1}}{(2 \pi i)^{m+1}} \int_{x}^{y} \frac{e^{\pi i\left(\omega t^{2}+2 k t\right)}}{(\omega t+k)^{2 m+2}} d t
\end{aligned}
$$


Thus,

$$
\begin{aligned}
\left(\sum_{k=-\infty}^{-n-1}\right. & \left.+\sum_{k=n+1}^{\infty}\right) \int_{-N}^{N} e^{2 \pi i \phi_{k}} d t-\left\{\sum_{k=-n}^{n-1} \int_{-\infty}^{-N}+\sum_{k=-n+1}^{n} \int_{N}^{\infty}\right\} e^{2 \pi i \phi_{k}} d t \\
& =e^{\pi i \omega N^{2}} \sum_{\substack{k=-\infty \\
k \neq-n}}^{\infty}\left\{\frac{1}{2 \pi i(\omega N+k)}+\sum_{\ell=1}^{m} \frac{(2 \ell-1) ! ! \omega^{\ell}}{(2 \pi i)^{\ell+1}(\omega N+k)^{2 \ell+1}}\right\} \\
& -e^{\pi i \omega N^{2}} \sum_{\substack{k=-\infty \\
k \neq+n}}^{\infty}\left\{\frac{1}{2 \pi i(-\omega N+k)}+\sum_{\ell=1}^{m} \frac{(2 \ell-1) ! ! \omega^{\ell}}{(2 \pi i)^{\ell+1}(-\omega N+k)^{2 \ell+1}}\right\} \\
& +I \\
& =S_{1}+S_{2}+I .
\end{aligned}
$$

Here, in accordance with the application of the Poisson sum formula, divergent sums of the form $\sum_{k=-\infty}^{\infty} 1 /(x+k)$ are considered in the sense

$$
\lim _{K \rightarrow \infty} \sum_{k=-K}^{K} 1 /(x+k)-1 / x=\lim _{K \rightarrow \infty} \sum_{k=1}^{K} 2 x /\left(x^{2}-k^{2}\right),
$$

which is defined and converges uniformly for $|x| \leq|\epsilon|<1 / 2$.

The first two terms, $S_{1}$ and $S_{2}$, are rearranged by exchanging the order of the two summations to give (we work with $S_{1}$ only; $S_{2}$ follows similarly):

$$
S_{1}=\frac{e^{\pi i \omega N^{2}}}{2 \pi i} \sum_{\substack{k=-\infty \\ k \neq-n}}^{\infty} \frac{1}{\omega N+k}+e^{\pi i \omega N^{2}} \sum_{\ell=1}^{m} \frac{(2 \ell-1) ! ! \omega^{\ell}}{(2 \pi i)^{\ell+1}} \sum_{\substack{k=-\infty \\ k \neq-n}}^{\infty} \frac{1}{(\omega N+k)^{2 \ell+1}} .
$$

We observe that

$$
\left.\frac{d^{2 \ell}}{d t^{2 \ell}} \sum_{\substack{k=-\infty \\ k \neq-n}}^{\infty} \frac{1}{\omega t+k}\right|_{t=N}=\left.(-1)^{2 \ell} \omega^{2 \ell}(2 \ell) ! \sum_{\substack{k=-\infty \\ k \neq-n}}^{\infty} \frac{1}{(\omega t+k)^{2 \ell+1}}\right|_{t=N}
$$

since the series can be differentiated term by term (this is obvious since, away from poles, convergence of series is guaranteed by comparison to $\left.\sum_{1}^{\infty} \frac{1}{k^{2}}\right)$. So, if we observe that

$$
\begin{aligned}
P V \sum_{\substack{k=-\infty \\
k \neq-n}}^{\infty}\left(\frac{1}{\omega N+k}\right) & =P V \sum_{k=-\infty}^{\infty}\left(\frac{1}{\omega N+k}\right)-\frac{1}{\omega N-n} \\
& =\pi \cot (\pi \epsilon)-\frac{1}{\epsilon} \equiv \psi(\epsilon)
\end{aligned}
$$

with $\psi(z)$ analytic for $|z|<1$, this term can be written compactly as

$$
\sum_{\substack{k=-\infty \\ k \neq-n}}^{\infty} \frac{1}{(\omega N+k)^{2 \ell+1}}=\left.\frac{1}{(2 \ell) !} \frac{d^{2 \ell}}{d s^{2 \ell}} \psi(s)\right|_{s=\epsilon} .
$$

Here we used the fact $|\omega N-n|=|\epsilon|<|\omega| / 2<1 / 2$, which follows from $N=[[n / \omega]]$.

Now

$$
S_{1}=\left.\frac{e^{\pi i \omega N^{2}}}{2 \pi i} \sum_{l=0}^{m} \frac{\omega^{\ell}}{(2 \pi i)^{\ell}(2 \ell) ! !} \frac{d^{2 \ell}}{d s^{2 \ell}} \psi(s)\right|_{s=\epsilon},
$$


and similarly

$$
S_{2}=-\left.\frac{e^{\pi i \omega N^{2}}}{2 \pi i} \sum_{\ell=0}^{m} \frac{\omega^{\ell}}{(2 \pi i)^{\ell}(2 \ell) ! !} \frac{d^{2 \ell}}{d s^{2 \ell}} \psi(s)\right|_{s=-\epsilon} .
$$

Since $\psi(s)$ and its derivatives of even order are odd functions of $s \in[-1 / 2,1 / 2]$, the proof of Lemma 2.3 is complete.

We note that this part of the proof closely follows Wilton [23]. The main difference here is in our restrictions on the limits of the sums. At this stage, Wilton carries out a single integration by parts and estimates the remainder by using a triangle inequality. In our case, by carefully summing to upper limits related to the Diophantine approximations to $\omega$, we can arrange, as we shall see, near cancellations in the remainder terms, which will allow us to arrive at the Diophantine error estimate.

Following the conclusion of the previous lemma, we see that in the expression

$$
\left|S(-N, N ; \omega)-\frac{e^{i \pi / 4}}{\sqrt{\omega}} S(-n, n ;-1 / \omega)\right| \leq 2\left|\frac{\epsilon}{\omega}\right|+\left|\sigma_{1}-\delta_{2}-\delta_{3}-I\right|+|I|
$$

we can easily estimate the second part of the bound, if we take into account the fact that $\left|\psi^{(2 \ell)}(s)\right|$ is increasing and convex in $[0,1 / 2]$, so that $\psi^{(2 l)}(\epsilon) \leq 2 \epsilon \psi^{(2 l)}(1 / 2)$.

The estimate is

$$
\left|\sigma_{1}-\delta_{2}-\delta_{3}-I\right| \leq\left(4 \sum_{\ell=0}^{m} \frac{|\omega|^{\ell}}{(2 \pi)^{\ell+1}(2 \ell) ! !}\left|\psi^{(2 \ell)}(1 / 2)\right|\right)|\epsilon| .
$$

For $m=1$ this gives

$$
\left|\sigma_{1}-\delta_{2}-\delta_{3}-I\right| \leq \frac{4}{\pi}\left(1+\frac{2 \omega}{\pi}\right)|\epsilon| \leq(1.273240+.810570 \omega)|\epsilon| .
$$

Clearly, the above expression is of the form $c_{1}|\epsilon|$ with $c_{1}$ some constant, independent of $n$ and $\omega$. We now show that the term $I$ obeys a similar Diophantine bound. First, we rewrite $I$ in a form suitable for estimation. We have

Lemma 2.4. If $K=2(2 m+1) ! ! \omega^{m+1} /(2 \pi i)^{m+1}$ and $\epsilon=\omega N-n$, then

$$
\begin{aligned}
I / K & =\sum_{p=1}^{\infty} \sum_{\ell=-n+1}^{n} \int_{-N}^{N} e^{i \pi\left[\omega t^{2}+2(\ell+2 p n) t\right]} \\
& \times\left\{\frac{1}{(\omega t+\ell+2 p n)^{2 m+2}}-\frac{e^{4 p i \pi \epsilon(t+p N)}}{(\omega t+\ell+2 p n+2 p \epsilon)^{2 m+2}}\right\} d t .
\end{aligned}
$$

Proof. Taking advantage of the invariance of the integrand in (30) under the change of variables $k \rightarrow-k, t \rightarrow-t$, we see that $I$ can be written as

$$
I=2 \frac{(2 m+1) ! ! \omega^{m+1}}{(2 \pi i)^{m+1}}\left\{\sum_{k=n+1}^{\infty} \int_{-N}^{N}-\sum_{k=-n+1}^{n} \int_{N}^{\infty}\right\} \frac{e^{\pi i\left(\omega t^{2}+2 k t\right)}}{(\omega t+k)^{2 m+2}} d t .
$$

We adjust the first sum $\sum_{n+1}^{\infty}$ by $k=\ell+2 n p(p=1,2, \ldots ;-n+1 \leq \ell \leq n)$, and we adjust the second integral $\int_{N}^{\infty}$ by $t=s+2 p N(p=1,2, \ldots ;-N \leq s \leq N)$. Renaming indices appropriately, we get the form claimed above.

If we let

$$
F_{n, p}(t ; \epsilon)=\frac{e^{4 \pi i \epsilon p(t+p N)}}{[\omega t+\ell+2 p n+2 p \epsilon]^{2 m+2}},
$$


we notice that for integers $\ell \in[-n+1, n]$ and $t \in[-N, N]$ the denominator is bounded away from zero. That all the integrals in $I$ have stationary phase points outside the domain of integration, of course, guaranteed this. The sum $\sum_{p=1}^{\infty} \int_{-N}^{N} F_{n, p}(t ; \epsilon)$ is absolutely, and hence uniformly, convergent. Thus we can apply the first theorem of the mean and differentiate term by term to find, for $\theta$ between 0 and $\epsilon$,

$$
\begin{aligned}
\left(\frac{I}{K}\right)= & \sum_{p=1}^{\infty} \sum_{l=-n+1}^{n} \int_{-N}^{N}\left(F_{n, p}(t ; 0)-F_{n, p}(t ; \epsilon)\right) d t \\
= & -\left.\epsilon \sum_{p=1}^{\infty} \sum_{l=-n+1}^{n} \int_{-N}^{N} \frac{\partial F_{n, p}(t ; s)}{\partial s}\right|_{s=\theta} d t \\
= & \sum_{p=1}^{\infty} \sum_{l=-n+1}^{n} \int_{-N}^{N} \epsilon\left\{\frac{4(m+1) p}{[\omega t+\ell+2 p n+2 p \theta]^{2 m+3}}\right. \\
& \left.-\frac{4 \pi i p(t+p N)}{[\omega t+\ell+2 p n+2 p \theta]^{2 m+2}}\right\} e^{4 \pi i \theta p(t+p N)} d t .
\end{aligned}
$$

Thus,

$$
\begin{aligned}
|I / K| \leq|\epsilon| \sum_{p=1}^{\infty} 4 p \sum_{\ell=-n+1}^{n} \int_{-N}^{N}\{ & \pi(t+p N)[\omega t+\ell+2 p(n+\theta)]^{-2 m-2} \\
& \left.+(m+1)[\omega t+\ell+2 p(n+\theta)]^{-2 m-3}\right\} d t .
\end{aligned}
$$

The sum converges for $m \geq 1$. Integrating, we find the following expression for $I / K$ :

$$
+\frac{p \pi}{(2 m+1) \omega^{2}}\left\{-\frac{[p(\omega N-2(n+\theta))-l]}{[\omega N+l+2 p(n+\theta)]^{(2 m+1)}}+\frac{[p(\omega N-2(n+\theta))-l]}{[-\omega N+l+2 p(n+\theta)]^{(2 m+1)}}\right\}
$$

$$
+\frac{p}{(2 m+2) \omega}\left\{-\frac{1}{[\omega N+l+2 p(n+\theta)]^{(2 m+2)}}+\frac{1}{[-\omega N+l+2 p(n+\theta)]^{(2 m+2)}}\right\} .
$$

Next we could put each pair of fractions enclosed in curly brackets over a common denominator. After cancellation in the numerators, one determines that these three terms are each as small as $c / p^{(2 m+1)}$ for some $c$ independent of $p$. Thus, $|I / K|$ is bounded by $|\epsilon| c$ for some $c$ independent of $p$ for any $m \geq 1$, and this establishes the existence of an upper bound for $C$ in our theorem. However, this would result in an overestimate. The bound claimed in Theorem 2.1 is found for $m=1$ by carefully balancing the terms in $I$. The details of this calculation are given in the appendix. 


\section{THE RENORMALIZATION MAP}

In the previous section we discussed how the summation of (15) in blocks leads to a new sum over a lesser range, characterized by a new parameter in the exponent (16):

$$
\left.\omega \rightarrow-\frac{1}{\omega} \quad(\bmod 2), \omega \in\right]-1,1[\backslash\{0\} .
$$

Hardy and Littlewood [11] utilized this mapping to give an estimate of the growth of (15) in terms of the entries in the Euclidean, or regular, continued fraction (abbreviated in the sequel by $r c f$ ) expansion of $\omega$. We write

$$
\omega=\frac{a_{1}}{b_{1}+} \frac{a_{2}}{b_{2}+} \ldots \equiv\left[\frac{a_{k}}{b_{k}}\right]_{k=1}^{\infty} \equiv\left[\frac{a_{1}}{b_{1}}, \cdots, \frac{a_{n}}{b_{n}}, \cdots\right] \equiv\left[\frac{a_{1}}{b_{1}}, \cdots, \frac{a_{n}}{b_{n}}, \omega_{n}\right]
$$

and we define the $n$-th convergent $\alpha_{n}$ and remainder $\omega_{n}$ by

$$
\alpha_{n} \equiv\left[\frac{a_{k}}{b_{k}}\right]_{k=1}^{n} \equiv \frac{p_{n}}{q_{n}}, \omega_{n} \equiv\left[\frac{a_{k}}{b_{k}}\right]_{k=n+1}^{\infty}
$$

The (relatively prime) integers $p_{k}\left(q_{k}\right)$ are the $k$-th convergent numerators (respectively, denominators) of the continued fraction representation of $\omega$. The rcf for $\omega \in]-1,1\left[\right.$ is found if we set $a_{k}=1, b_{k} \in \mathbb{Z}^{+}$, for which we write

$$
\omega=\frac{1}{b_{1}+} \frac{1}{b_{2}+} \cdots \frac{1}{b_{n}+\omega_{n}} \equiv\left[b_{k}\right]_{k=1}^{\infty} \equiv\left[b_{1}, \cdots, b_{k}, \cdots\right] \equiv\left[b_{1}, \cdots, b_{n-1}, b_{n}+\omega_{n}\right] \text {. }
$$

Clearly, convergence of the above expressions needs to be established before we can employ the equality signs. However that is assured for rcf, for which optimal two-sided convergence of the partial convergents $\alpha_{n}$ to the irrational $\omega$ is well known [12]. Other expansion algorithms are possible. In our discussion we shall employ even continued fractions $(e c f)$, for which $a_{k}=1, b_{k} \in 2 \mathbb{Z}$. Convergence of ecf is proved below. In the sequel, we shall freely interchange between a number and its (convergent) continued fraction expansion.

In terms of the rcf, applying (39) to $\omega$ we have, if $\omega^{(k)}$ is the $k$-th iterate,

$$
\begin{aligned}
\omega \equiv \omega^{(0)} & =\frac{1}{b_{1}+\omega_{1}} \rightarrow-\frac{1}{\omega^{(0)}} \quad(\bmod 2)=\omega^{(1)} \\
& =\left(-b_{1}-\omega_{1}\right) \quad(\bmod 2)= \begin{cases}-\omega_{1}, & \text { if } b_{1} \text { is even, } \\
1-\omega_{1}, & \text { if } b_{1} \text { is odd }\end{cases}
\end{aligned}
$$

It is not too difficult to devise simple transformation rules to produce the rcf of $1-\omega_{k}$ from that of $\omega_{k}$, and thus construct a recursive algorithm to compute the successive iterates of the map. However, the orbit of the mapping is unknown even if the rcf expansion of $\omega$ is known in advance. The ergodic properties of (39) were explored more recently by Berry and Goldberg [2], using the rcf.

As we showed in [8], the natural representation which unfolds the orbits of (39) involves expressing the irrational $\omega$ in terms of even continued fractions, namely continued fractions with even (positive or negative) entries. Thus, consider the expansion

$$
-1<\omega=\left[2 d_{k}\right]_{k=1}^{\infty}<+1
$$

with $d_{j} \in \mathbb{Z}, j=1,2,3, \ldots$ Assuming the first $k$ entries are known, we have

$$
\omega \equiv \omega_{0}=\left[2 d_{1}, 2 d_{2}, \ldots, 2 d_{k}+\omega_{k}\right]
$$


with $-1<\omega_{k} \leq 1$. The $k+1$-st entry is found as follows: (i) If $\omega_{k}=0$ or $\omega_{k}=1$ the fraction terminates. (ii) Else, $1<\frac{1}{\left|\omega_{k}\right|}<\infty$ and we let $r=\left\lfloor\frac{1}{\omega_{k}}\right\rfloor$. If $r$ is even, set $d_{k+1}=r / 2$; then $0<\omega_{k+1}=1 / \omega_{k}-r<1$. Alternatively, with odd $r$, set $d_{k+1}=(r+1) / 2$ and $-1<\omega_{k+1}=1 / \omega_{k}-r-1<0$. We observe that successive remainders are related via the Möbius transformation,

$$
\omega_{k-1}=\frac{1}{2 d_{k}+\omega_{k}} .
$$

Thus, for rational $\omega=p / q$, when the fraction terminates and all the partial remainders are rational as well, we have that

$$
\omega_{k-1} \equiv \frac{P_{k-1}}{Q_{k-1}}=\frac{1}{2 d_{k}+\omega_{k}}=\frac{Q_{k}}{2 d_{k} Q_{k}+P_{k}} .
$$

Since the fractions $P_{k} / Q_{k}$ are assumed irreducible (and $P_{k} \leq Q_{k}$ ), we deduce that

$$
Q_{k}=P_{k-1}, \quad P_{k}=Q_{k-1}-2 d_{k} P_{k-1} .
$$

The orbit of the map (39) is found simply from the remainders $\omega_{k}$ in (42),

$$
\begin{aligned}
\omega^{(k-1)} & =(-1)^{k-1} \omega_{k-1}=(-1)^{k-1} \frac{1}{2 d_{k}+\omega_{k}} \\
& \rightarrow(-1)^{k}\left(2 d_{k}+\omega_{k}\right) \quad(\bmod 2)=(-1)^{k} \omega_{k}=\omega^{(k)} .
\end{aligned}
$$

It follows from (43) that the action of the map (39) on rationals has the property that if

$$
\frac{p_{k-1}}{q_{k-1}} \rightarrow \frac{p_{k}}{q_{k}}=-\frac{q_{k-1}}{p_{k-1}} \quad(\bmod 2),
$$

then

$$
p_{k} q_{k} \quad(\bmod 2)=p_{k-1} q_{k-1} \quad(\bmod 2) .
$$

This, in turn, implies that the orbit of the rational $p / q$ will end in a zero or one according to the parity of the product $p q$.

The following lemma gives the relationship between the even and Euclidean continued fraction expansions of an irrational $\omega$.

Lemma 3.1. Suppose that the Euclidean continued fraction for an irrational number $\omega=\omega_{0}$ is given by

$$
\omega_{0}=\left[a, b, c+\omega_{3}\right], a, b, c \in \mathbb{Z}^{+}, 0<\omega_{3}<1 .
$$

Then $\omega$ has the equivalent continued fraction

$$
\omega=[a+1, \underbrace{-2,2, \cdots,-(-1)^{b} 2}_{b-1 \text { terms }},(-1)^{b}\left(c+1+\omega_{3}\right)] .
$$

Proof. We write

$$
\omega_{0}=\frac{1}{a+\omega_{1}}=\left[a+\omega_{1}\right]=\left[a, b+\omega_{2}\right]=\left[a, b, c+\omega_{3}\right] .
$$

Now,

$$
1-\omega_{1}=1-\frac{1}{b+\omega_{2}}=\frac{b-1+\omega_{2}}{b+\omega_{2}}=\left[1, b-1+\omega_{2}\right]=\left[1, b-1, c+\omega_{3}\right] .
$$


Note that for $b=1$ we get $1-\omega_{1}=\left[1,0, c+\omega_{3}\right]=\left[1+c+\omega_{3}\right]$, since $[\cdots, x, 0, y, \cdots]=$ $[\cdots, x+y, \cdots]$ in general. Using the obvious identities

$$
-[k, l, m, \cdots]=[-k,-l,-m, \cdots] \quad \text { and } \quad[a,[b, c, \cdots]]=[a, b, c, \cdots],
$$

we find that

$$
\omega=\left[a+\omega_{1}\right]=\left[a+1-\left(1-\omega_{1}\right)\right]=\left[a+1,-1,-\left(b-1+\omega_{2}\right)\right] .
$$

Applying (47) to the continued fraction for $1-\omega_{1}$ in (46) in turn, we get

$$
1-\omega_{1}=\left[1, b-1+\omega_{2}\right]=\left[2,-1,-\left(b-2+\omega_{2}\right)\right]=\left[2,-2,1,\left(b-3+\omega_{2}\right)\right], \quad \text { etc. }
$$

Clearly, this process can be repeated $b-1$ times. Incorporating the result in the continued fraction for $\omega$, we find that

$$
\omega=\left[a+1,-\left[1, b-1+\omega_{2}\right]\right]=\ldots=[a+1, \underbrace{-2,2, \cdots,-(-1)^{b} 2}_{b-1 \text { terms }},(-1)^{b}\left(1+c+\omega_{3}\right)],
$$

which completes the proof.

With the use of the above lemma we can now give an algorithm for the conversion of an ref to an ecf. We have

Corollary 3.1. Given an irrational $\omega \in]-1,+1[\backslash\{0\}$ and the rcf

$$
\omega=\left[b_{1}, b_{2}, \ldots, b_{k}, b_{k+1}, b_{k+2}+\omega_{k+2}\right],
$$

where the first odd entry occurs at the $k$-th place (i.e. $b_{i}$ is even, $i=1, \ldots, k-1$, while $b_{k}$ is odd). Then there is an equivalent continued fraction, in which the first odd entry cannot occur before the $k+b_{k+1}$-st place (and which may have negative entries), namely

$$
\omega=[b_{1}, b_{2}, \ldots, b_{k}+1, \underbrace{-2,2, \cdots,-(-1)^{b_{k+1}}}_{b_{k+1}-1 \text { terms }},(-1)^{b_{k+1}}\left(b_{k+2}+1+\omega_{k+2}\right)] .
$$

In this new fraction, the $k$-th entry has been increased by one, while the $k+1$-st entry has been replaced by a sequence of $b_{k+1}-1$ 2's with alternating signs. The first possibly odd entry may only occur past that sequence.

This recursive algorithm for constructing the ecf from the known rcf of a number is of course no different (apart from signs) from the algorithm alluded to earlier for computing the orbits of (39) from the $\operatorname{rcf}$ of $\omega$.

The convergence of fractions of form (41) is guaranteed.

Lemma 3.2. Ecf of form (41) always converge to some number $w \in]-1,+1[$, so that, if $w_{n}$ is the $n$-th convergent then $\left|w-w_{n}\right|<1 /(2 n+1)$.

Proof. Let

$$
C \equiv \frac{1}{2 \beta_{1}+2 \beta_{2}+} \cdots,
$$

where $\beta_{k} \in \mathbb{Z} \backslash\{0\}$. This can be transformed via an equivalence transformation to the form

$$
C \equiv \frac{c_{1} / 2}{c_{1} 2 \beta_{1}+} \frac{c_{1} c_{2}}{c_{2} 2 \beta_{2}+} \ldots \frac{c_{n-1} c_{n}}{c_{n} 2 \beta_{n}+} \ldots
$$


which for $c_{n}=1 /\left(2 \beta_{n}\right)$ becomes

$$
C \equiv \frac{1 /\left(4 \beta_{1}\right)}{1+} \ldots \frac{1 /\left(4 \beta_{n-1} \beta_{n}\right)}{1+} \ldots .
$$

The assertion then follows from Worpitzky's theorem [12].

As this lemma suggests, ecf always converge, but in some cases the convergence might be only linear. However, it is evident from the form of (48) that the remainders of the rcf and ecf are closely related, so that, if the ecf is truncated at the end of any of the possible long alternating sequences of \pm 2 's, it will provide similar partial convergents as the rcf, sharing some of the same optimality properties.

We consider now the question of successive renormalizations of the sum (15), that is, of successive applications of the approximate functional formula (16). The connection with Lehmer's result [16] is instructive in this context; in his discussion of "incomplete" gauss sums, he observes that the basic building blocks of the pattern for a special case (which characterizes the first step in the renormalization process) are given by the following four cases:

$$
\sum_{k=0}^{N}{ }^{i \pi k^{2}\left(\frac{2}{N}\right)}=\left\{\begin{array}{cl}
(1+i) \sqrt{N}, & N=4 l \\
\sqrt{N}, & N=4 l+1, \\
0, & N=4 l+2, \\
i \sqrt{N}, & N=4 k+3,
\end{array}\right.
$$

The pattern corresponding to each one of these cases is shown in Figure 4 on page 633. Now, in our irrational-exponent sums, the ecf of the exponent $\omega$ unfolds the orbits of the renormalization map. But what is the appearance of the basic building blocks ("0-level spirals") of the graph of the partial sums? It is clear that truncation in this form does not reveal the structure of the graph (i.e. the rational number that results when the ecf for the irrational $\omega$ is truncated is not the appropriate exponent that governs the graph). But defining $c_{2 k}=1, c_{2 k-1}=2$, we introduce the equivalence transformation $a_{k}=1 \rightarrow c_{k-1} c_{k} a_{k}=2, b_{k}=2 d_{k} \rightarrow 2 c_{k} d_{k}$, to obtain the equivalent fraction

$$
\omega=\frac{2}{4 d_{1}+} \frac{2}{2 d_{2}+} \frac{2}{4 d_{3}+} \frac{2}{2 d_{4}+} \ldots<+1 .
$$

We see that truncation at the second level,

$$
\omega \approx \frac{2}{4 d_{1}+\frac{2}{2 d_{2}+}}
$$

reveals precisely the building blocks: $\left|d_{2}\right|>1$ : spirals of type $1 ; d_{2}= \pm 1$ : cases 2,4 . After the first renormalization,

$$
\omega \rightarrow-\frac{1}{\omega} \quad(\bmod 2)=\frac{1}{2 d_{2}+\cdots} .
$$

Still, we must return to the original even representation for the clearest interpretation of the renormalization, while using the above equivalent form to classify the patterns. Naturally, any $d_{k}= \pm 1$ contributes twists of type 2 , 4 , while other values contribute connections of type 1 . An easy check shows that (49) can be deduced from (16) by properly choosing a rational $\omega$.

We find that for general $\omega$, successive applications of the renormalization formula (14) do not preserve the Diophantine property of the error for arbitrary N. However, for a certain class of quadratic irrationals there are integers $N_{l}$ (forming 
a subsequence of the sequence of convergent denominators of $\omega$ ) such that if we consider the sum in (15) with upper limit $N=N_{l}$ and lower limit 0 , then it is possible to carry out successive renormalizations while preserving the Diophantine character of the error term. More specifically, if $\omega$ has the form

$$
\omega_{\ell}^{(1)}=\frac{1}{2 \ell+} \frac{1}{(-2) \ell+} \ldots, \quad 1<\ell \in \mathbb{Z}^{+},
$$

namely, if $\omega$ is a fixed point of the renormalization map, or the form

$$
\omega_{\ell}^{(2)}=\frac{1}{2 \ell+} \frac{1}{2 \ell+} \ldots, \quad \ell \in \mathbb{Z}^{+},
$$

namely, if $\omega$ is such a periodic point of the renormalization map of period 2 , then we have

Lemma 3.3. If $\omega$ is a (quadratic) irrational whose ecf has the form (50) or (51) and $p_{n-1} / q_{n-1}$ and $p_{n} / q_{n}$ are its partial convergent approximations of order $n-1$ and $n$ respectively, then $p_{n}=q_{n-1}$.

Proof. For $\omega_{\ell}^{(2)}$ we have

$$
\begin{aligned}
p_{1}=1, & q_{1}=2 \ell, \\
p_{2}=2 \ell, & q_{2}=4 \ell^{2}+1, \\
\cdots, & \cdots, \\
p_{n+1}=2 \ell p_{n}+p_{n-1}, & q_{n+1}=2 \ell q_{n}+q_{n-1} ;
\end{aligned}
$$

while for $\omega_{\ell}^{(1)}$, similarly, we have

$$
\begin{aligned}
p_{1}=1, & q_{1}=2 \ell, \\
p_{2}=2 \ell, & q_{2}=4 \ell^{2}-1, \\
\ldots, & \cdots, \\
p_{n+1}=2 \ell p_{n}+(-1)^{n} p_{n-1}, & q_{n+1}=2 \ell q_{n}+(-1)^{n} q_{n-1},
\end{aligned}
$$

and the claim follows by induction in either case.

Remark 3.1. It is easy to see that the periodic orbits of period $k>1$ are given by

$$
\omega^{(k)}=\frac{1}{\left(2 a_{1}\right)+} \cdots \frac{1}{2 a_{k}+(-1)^{k} \omega^{(k)}} \cdots
$$

An example of partial sums for a periodic point of period 3 is shown in Figure 2 (with $0 \leq N \leq 90000$ ).

The following obvious lemma defines the self-similar patterns under renormalization:

Lemma 3.4. The fixed points of the renormalization map,

$$
\omega_{\ell}^{(1)}=\frac{1}{2 \ell+} \frac{1}{(-2) \ell+} \ldots=\ell-\sqrt{\ell^{2}-1}, \quad 1<\ell \in \mathbb{Z}^{+},
$$

have the classical continuous fraction

$$
\omega_{\ell}^{(1)}=\frac{1}{(2 \ell-1)+} \frac{1}{1+} \frac{1}{(2 \ell-2)+} \frac{1}{1+} \frac{1}{(2 \ell-2)+} \ldots,
$$




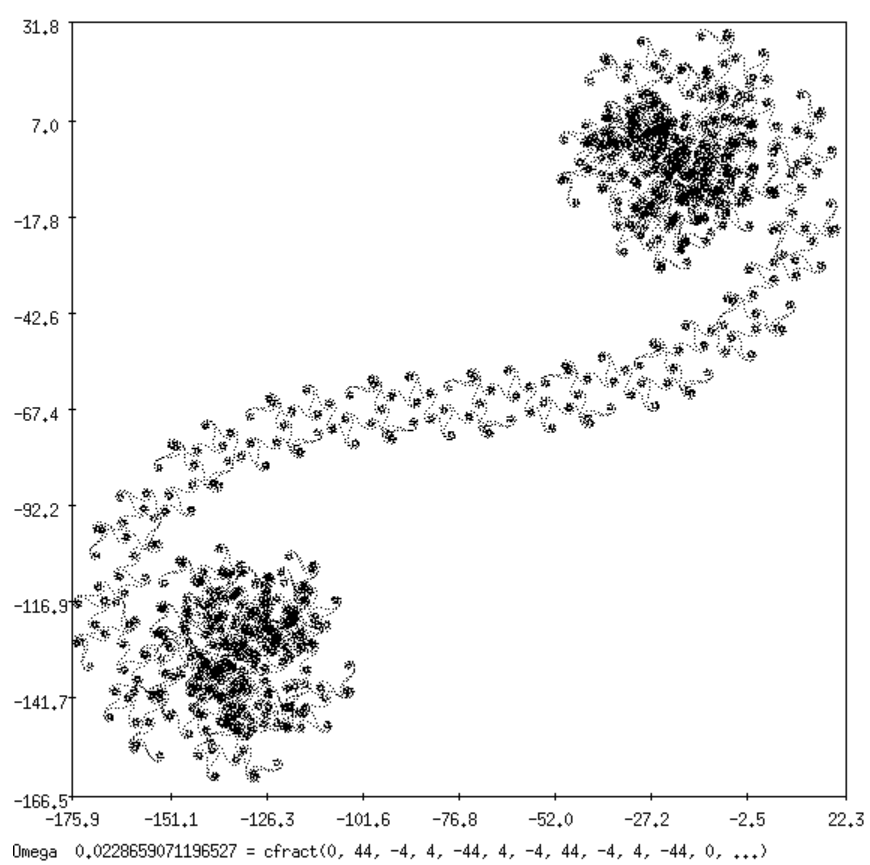

(a)

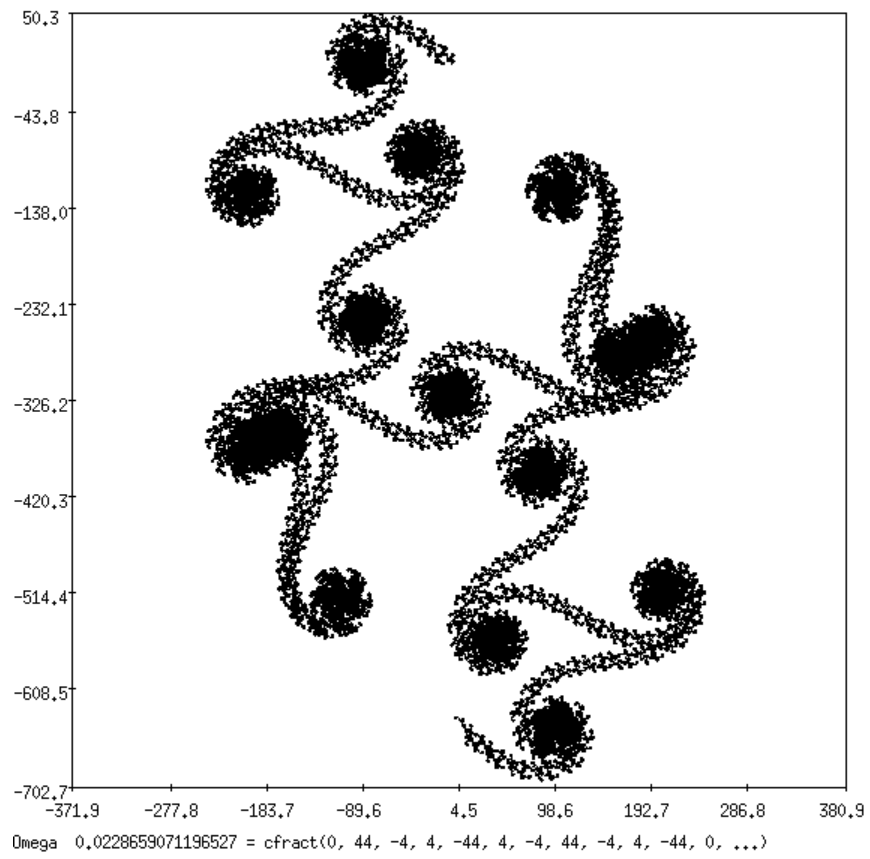

(b)

Figure 2. A period three pattern; top shows 4 levels $(12,000<$ $n<44,000)$ while bottom shows 6 levels $(N=428,000)$. 


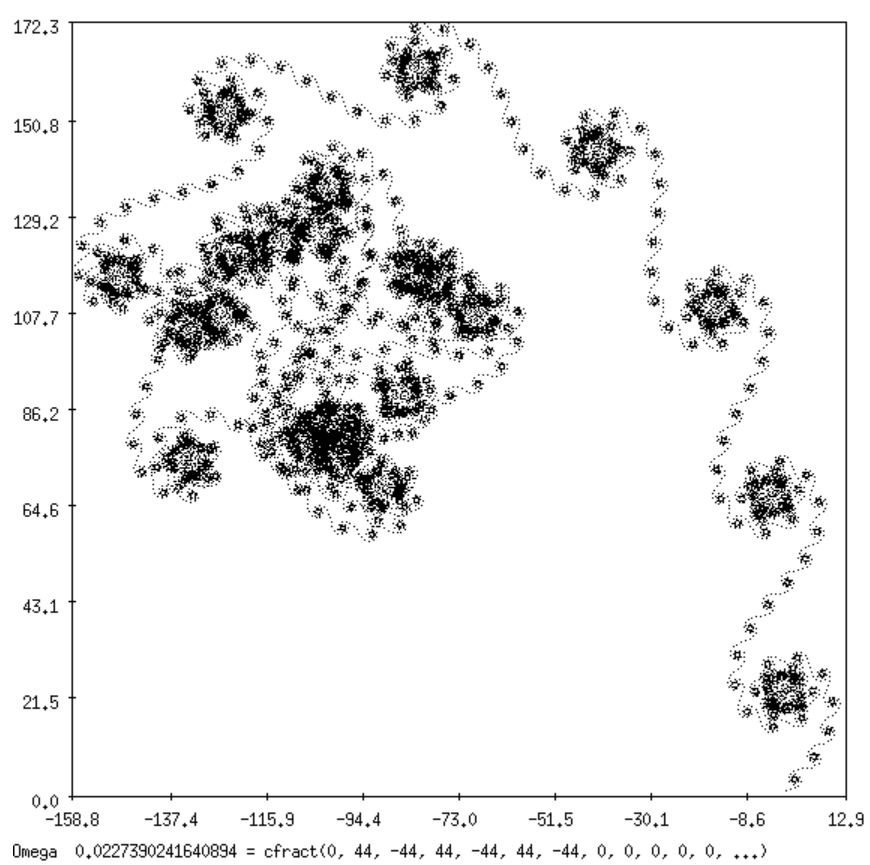

(a)

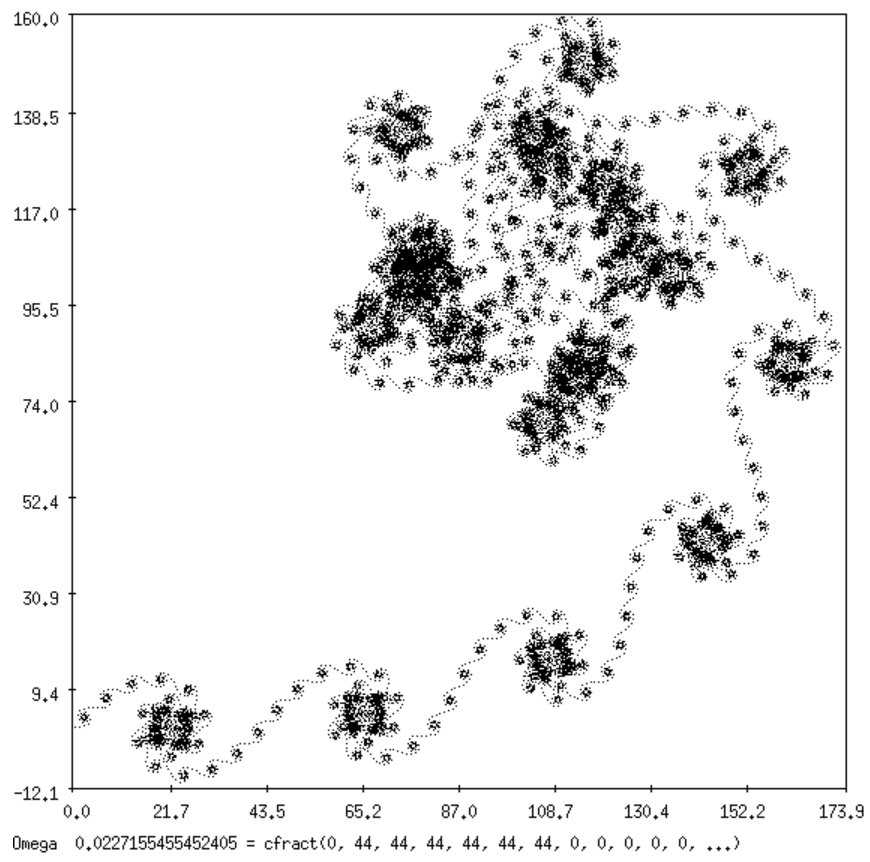

(b)

FiguRE 3. The selfsimilar and anti-selfsimilar spirals for $l=22$, $n=44000$ 


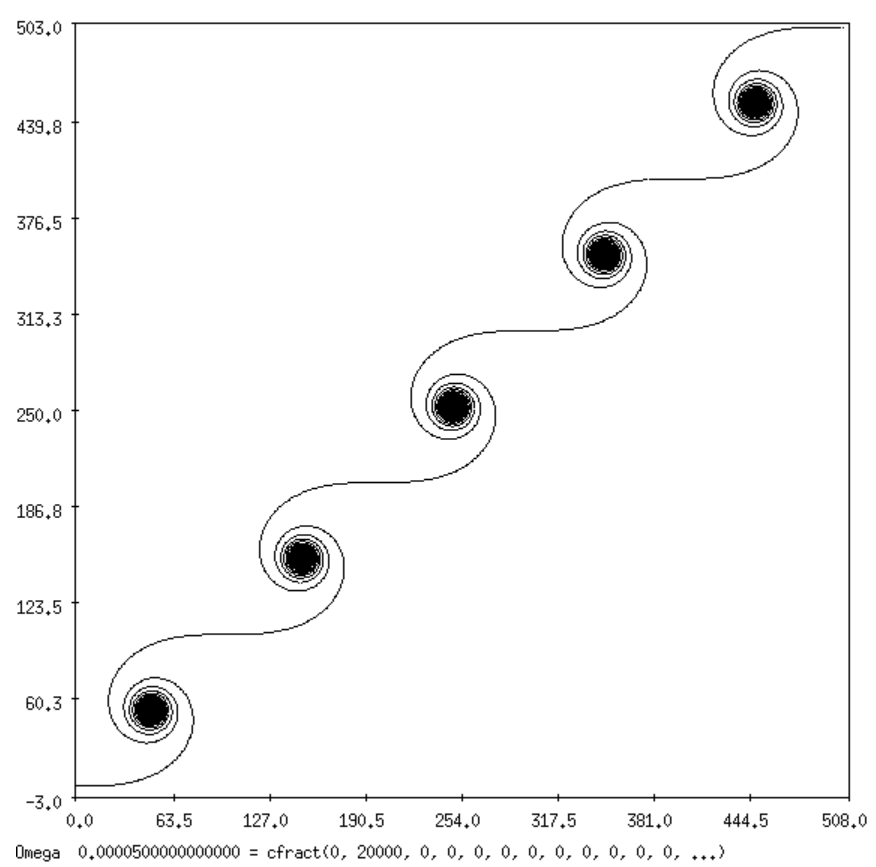

(a), $N=40,000$

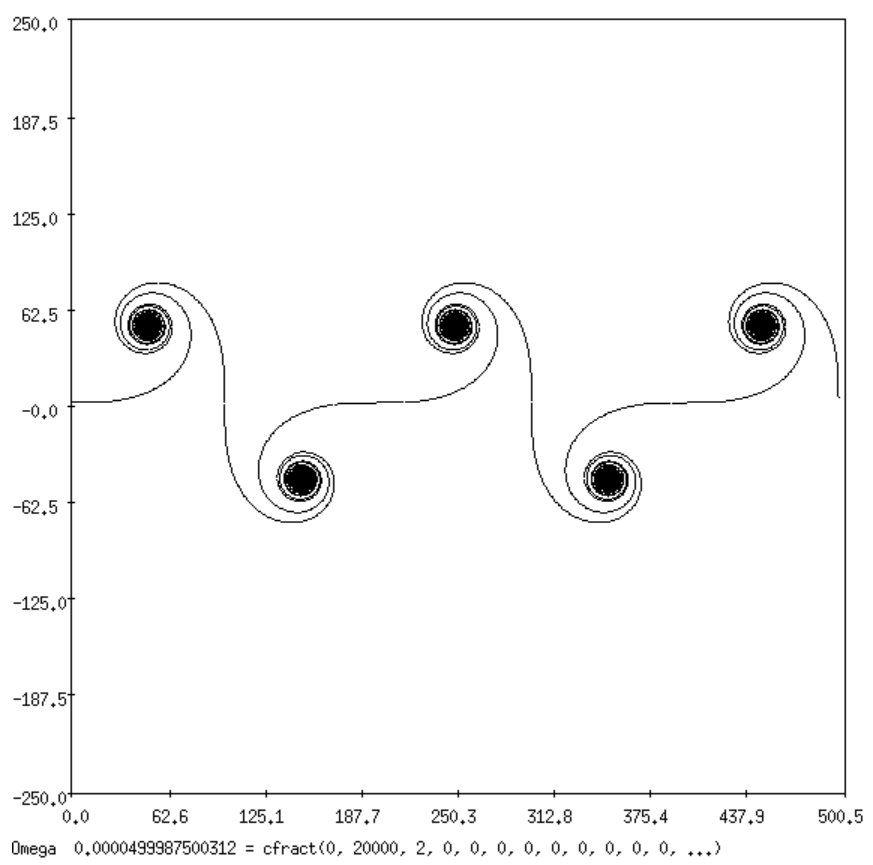

(b), $N=40,001$

Figure 4. The cases in (49). In each figure, 100,000 points were plotted 


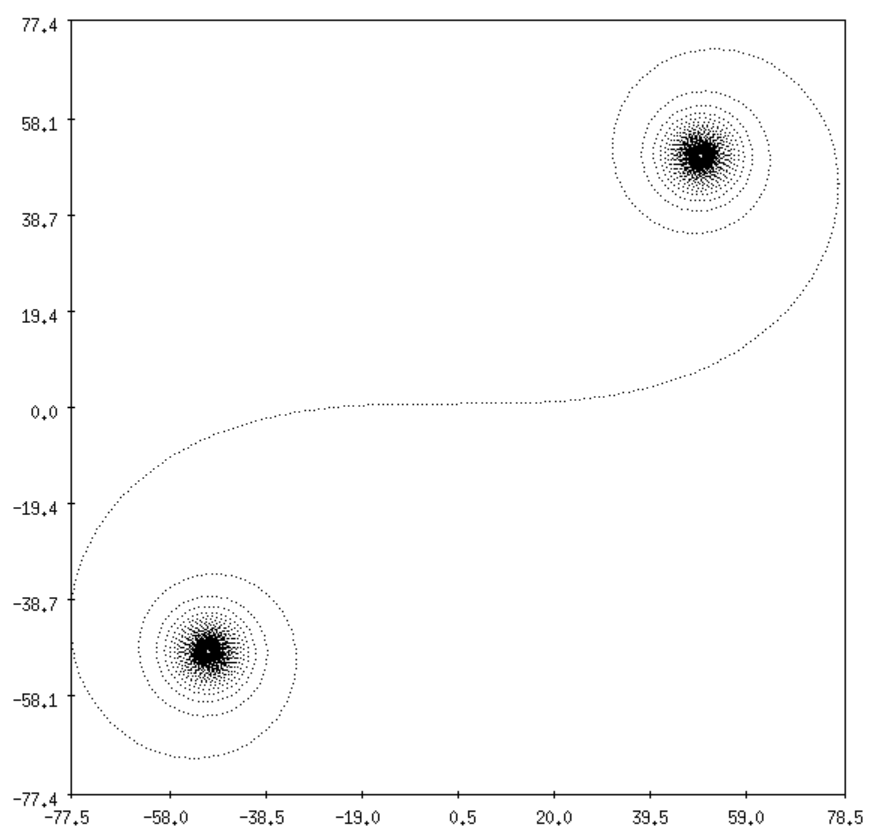

Omega $0,0000499975001250=\operatorname{cfract}(0,20000,2,0,0,0,0,0,0,0,0,0, \ldots)$

(c), $N=40,0002$

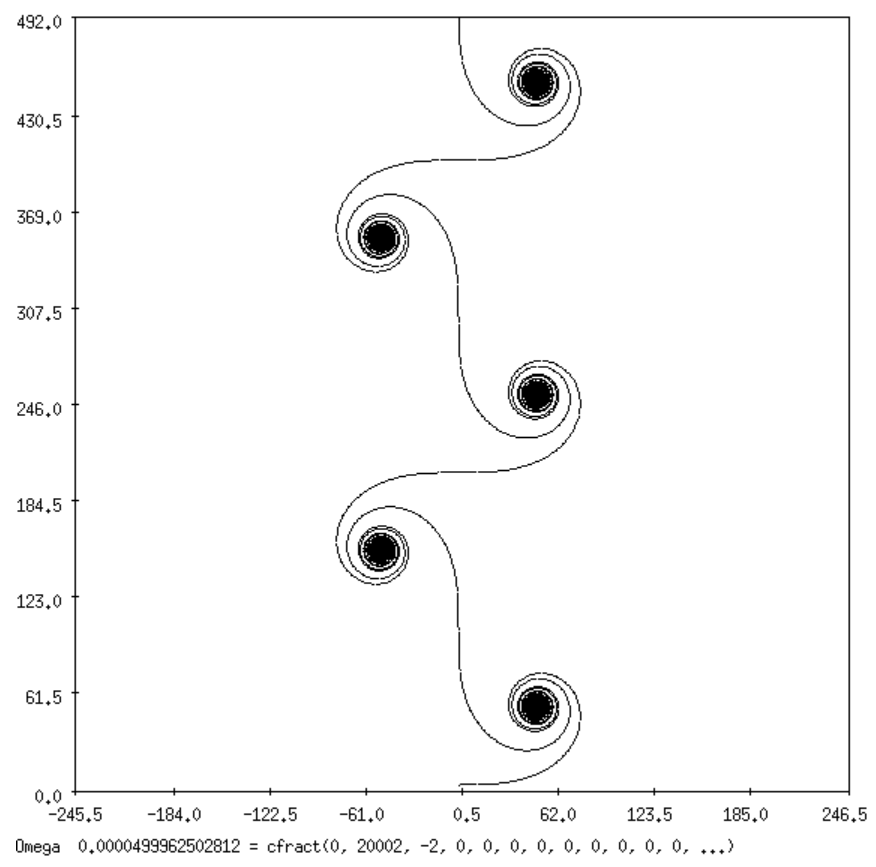

(d), $N=40,0003$

Figure 4. (continued) 


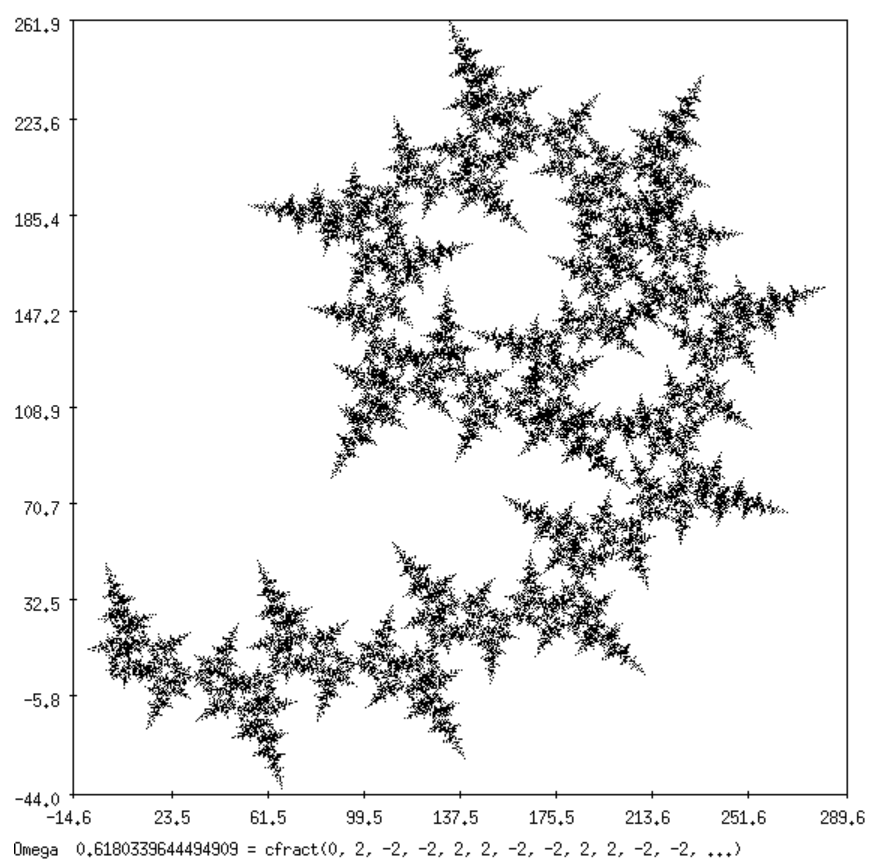

(a)

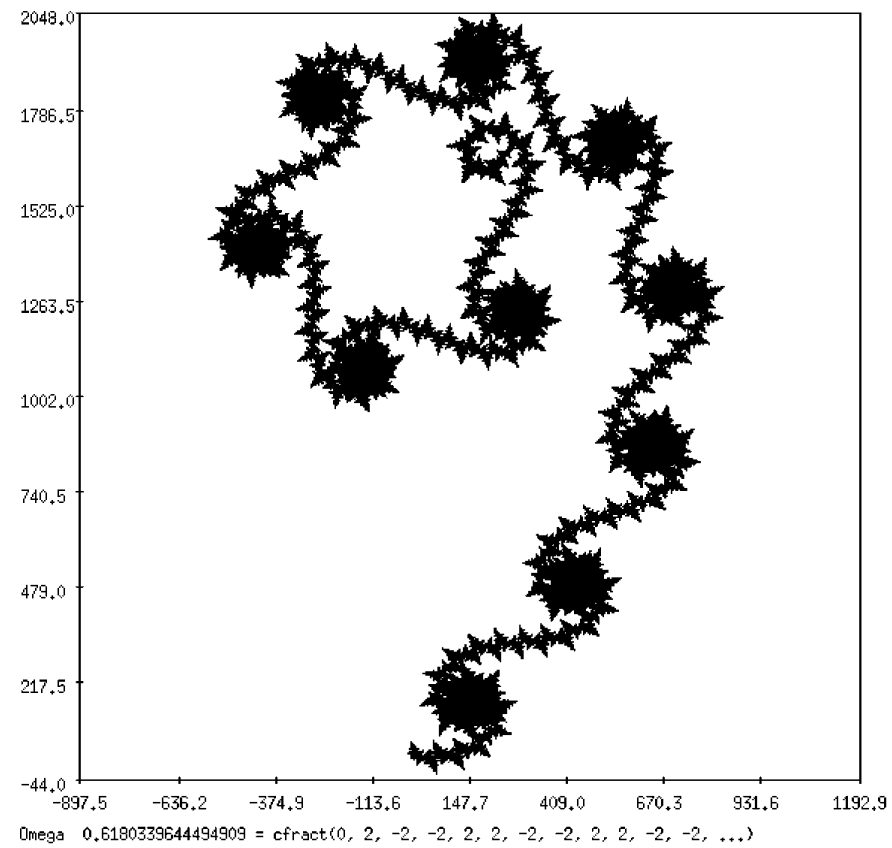

(b)

FiguRE 5. The graph for $\omega=\frac{1}{2} \frac{1}{-2} \frac{1}{-2} \frac{1}{2} \frac{1}{2} \frac{1}{-2} \frac{1}{-2} \frac{1}{2} \frac{1}{2} \frac{1}{-2} \frac{1}{-2} \frac{1}{2} \frac{1}{44} \frac{2}{-44} \ldots$ (top) 46000 points, (bottom) 1900000 points 
and the convergent numerators and denominators $P_{k}$ and $Q_{k}$, respectively, are given in terms of the $p_{n}, q_{n}$ defined in the proof of Lemma 3.1 by

$$
\begin{aligned}
P_{2 n}=p_{n}, & Q_{2 n}=q_{n}, \\
P_{2 n-1}=p_{n}-p_{n-1}, & Q_{2 n-1}=q_{n}-q_{n-1} .
\end{aligned}
$$

Note 1. Spirals of spirals are obtained either way. They differ only by one spiral of the lower order. The renormalizable one has $\epsilon_{n} \equiv \omega N-n>0\left(\epsilon_{n}<0\right)$ depending on whether the sequence is even (self-similar) or odd (anti-selfsimilar). Examples, with $\ell=22$ are shown in Figure 3a (self-similar) and Figure 3b (anti-selfsimilar), each with 44,000 points.

Thus it is seen that for the fixed points of the renormalization map, the sequence of partial convergents of the ecf is the even subsequence of the sequence of the partial convergents of the rcf. Hence it provides a monotonically increasing sequence of rational approximations of $\omega_{\ell}^{(1)}$ that satisfy the property

$$
\left|\omega_{\ell}^{(1)}-\frac{p_{n}}{q_{n}}\right|=\left|\omega_{\ell}^{(1)}-\frac{P_{2 n}}{Q_{2 n}}\right|<\frac{C}{Q_{2 n-1} Q_{2 n}}=\frac{C}{q_{n}\left(q_{n}-q_{n-1}\right)} .
$$

For the periodic points (49) of order two

$$
\omega_{\ell}^{(2)}=\frac{1}{2 \ell+} \frac{1}{2 \ell+} \cdots=\sqrt{\ell^{2}+1}-\ell, \quad 1<\ell \in \mathbb{Z}^{+} ;
$$

the entries are positive and the ecf coincides with the rcf. Repeated renormalizations can be also carried out in general, if we choose upper limits from the even convergent denominators of $\omega$. We state the rational case here. The extension to irrationals proceeds along similar lines. We have

Theorem 3.1. Consider the rational number $\omega=p / q, p q=0(\bmod 2)$. Let $\omega_{l}=$ $P_{l} / Q_{l}=\Phi_{k=l+1}^{n} \frac{-1}{2 d_{k}}, l=0, \cdots, n-1$, with $\omega_{0} \equiv \omega$ and $\left(P_{l}, Q_{l}\right)=1$. Then

$$
\begin{aligned}
\sum_{k=0}^{Q_{0}}{ }^{\prime} e^{i \pi \omega_{0} k^{2}} & =\left(\frac{-1}{\omega_{0}}\right)^{1 / 2} \sum_{k=0}^{Q_{1}}{ }^{\prime} e^{i \pi \omega_{1} k^{2}}=\cdots=\left(\frac{(-1)^{m}}{\omega_{0} \cdots \omega_{m-1}}\right)^{1 / 2} \sum_{k=0}^{Q_{m}}{ }^{\prime i \pi \omega_{m} k^{2}} \\
& =\cdots=\left(\frac{(-1)^{n}}{\omega_{0} \cdots \omega_{n-1}}\right)^{1 / 2} .
\end{aligned}
$$

Proof. Apply the Cauchy-Kronecker formula (4) repeatedly, taking advantage of (43) and the fact that $Q_{l+1}=P_{l}$. Note that the numerators are chosen here as $(-1)$ 's. This form is more convenient for the application of the map (39) since $\omega^{(k)}=\omega_{k}$; an equivalence transformation $a_{k} \rightarrow c_{k-1} c_{k} a_{k}, b_{k} \rightarrow c_{k} b_{k}$ with $c_{2 k}=-1$, $c_{2 k+1}=1$ reduces this to the previously used form (41).

This type of formula was of course known to Hardy and Littlewood; however, the use of ecf simplifies its statement dramatically.

Finally, we note that the behavior of the partial sums (9) in the complex plane as $N \rightarrow \infty$ is intermittent, in that they are governed by successively higher order entries in the continued fraction expansion of $\omega$. This means that as $N$ increases one may observe ordered behavior becoming disordered or the reverse. However, in accord with our main result, Theorem 2.1, the renormalization may proceed with ever increasing accuracy as $n \rightarrow \infty$ over successive convergent denominators of $\omega$. An example of initially disordered behavior which becomes increasingly more ordered 
as ever larger entries of the continued fraction are revealed by the renormalization map for increasing $N$ is shown in Figure $5(\mathrm{a}, \mathrm{b})$.

\section{ACKNOWLEDGEMENTS}

We would like to thank the referees for helpful suggestions. We would also like to thank Charles Troup for his help with the graphics. Portions of this work were done while the first author was a visiting professor at the Department of Mathematics of SUNY at Buffalo.

\section{Appendix A. The constant $C$ in the Diophantine inequality}

We introduce the notation

$$
\begin{gathered}
z=2(n+\theta), \quad x_{l}=\frac{l+\epsilon}{z}, \\
y_{1}=\frac{2 n+\epsilon}{z}=x_{2 n}, \quad y_{2}=\frac{2 n+2 \theta-\epsilon}{z}=1-\frac{\epsilon}{z}=1-x_{0},
\end{gathered}
$$

and rewrite (36)-(38), after some rearranging of the sums, as

$$
\left|\frac{I}{\epsilon}\right| \leq A_{1}+A_{2}
$$

with

(60)

$$
\begin{aligned}
A_{1} & =\frac{1}{\pi z^{2}} \sum_{l=1}^{2 n-1}\left(\sum_{p=-\infty}^{\infty} \frac{-\left(p+x_{l}\right)+x_{l}}{\left(p+x_{l}\right)^{2}}\right) \\
& +\frac{3 \omega}{2 \pi^{2} z^{4}} \sum_{l=1}^{2 n-1}\left(\sum_{p=-\infty}^{\infty} \frac{-\left(p+x_{l}\right)+x_{l}}{\left(p+x_{l}\right)^{4}}\right) \\
& +2 \frac{(n+\epsilon)}{\pi z^{3}} \sum_{l=1}^{2 n-1}\left(\sum_{p=-\infty}^{\infty}-\frac{\left(p+x_{l}\right)^{2}+\left(1-2 x_{l}\right)\left(p+x_{l}\right)+x_{l}\left(x_{l}-1\right)}{\left(p+x_{l}\right)^{3}}\right)
\end{aligned}
$$

and

(61)

$$
\begin{aligned}
& A_{2}=\frac{1}{\pi z^{2}} \sum_{p=0}^{\infty}\left(\frac{-\left(p+y_{1}\right)+y_{1}}{\left(p+y_{1}\right)^{2}}+\frac{\left(p+y_{2}\right)+1-y_{2}}{\left(p+y_{2}\right)^{2}}\right) \\
&+\frac{3 \omega}{2 \pi^{2} z^{4}} \sum_{p=0}^{\infty}\left(\frac{-\left(p+y_{1}\right)+y_{1}}{\left(p+y_{1}\right)^{4}}+\frac{\left(p+y_{2}\right)+1-y_{2}}{\left(p+y_{2}\right)^{4}}\right) \\
&+2 \frac{(n+\epsilon)}{\pi z^{3}} \sum_{p=0}^{\infty}\left(-\frac{\left(p+y_{1}\right)^{2}+\left(1-2 y_{1}\right)\left(p+y_{1}\right)+y_{1}\left(y_{1}-1\right)}{\left(p+y_{1}\right)^{3}}\right. \\
&\left.+\frac{\left(p+y_{2}\right)^{2}+\left(1-2 y_{2}\right)\left(p+y_{2}\right)+y_{2}\left(y_{2}-1\right)}{\left(p+y_{2}\right)^{3}}\right) .
\end{aligned}
$$

Estimate for $A_{1}$. We introduce the functions

$$
f_{k}(x)=\sum_{p=-\infty}^{\infty}(p+x)^{-k}, \quad f_{1}(x)=\sum_{p=-\infty}^{\infty}(p+x)^{-1}=\pi \cot (\pi x),
$$


(also, $f_{2}=\pi^{2} \csc ^{2}(\pi x), f_{3}=\pi^{3} \cot (\pi x) \csc ^{2}(\pi x), f_{4}=\pi^{4} \csc ^{2}(\pi x)\left[\frac{1+2 \cot ^{2}(\pi x)}{3}\right]$ ),

$$
\begin{gathered}
\phi_{k}(x)=x f_{k}(x)-f_{k-1}(x), \quad \phi_{1}(x)=x f_{1}(x), \\
q_{k}(x)=(1-x) \phi_{k}(x)+\phi_{k-1}(x), \quad q_{1}=(1-x) \phi_{1}(x) .
\end{gathered}
$$

These satisfy ( $g$ stands for any of $f, \phi$ or $q$ ):

$$
g_{k}^{\prime}=-k g_{k+1} \text {. }
$$

The $\phi_{k}, q_{k}$ are analytic at $x=0$. Using these properties and the fact that the $f_{n}$ are even (odd) about $x=1 / 2$ whenever $n$ is even (odd), and $f_{2 n}>0$ on $(0,1)$ while $f_{2 n+1}>0$ on $(0,1 / 2)$, it follows easily that $\phi_{2}$ and $\phi_{4}$ are increasing convex functions on $[0,1)$, while $q_{3}$ is odd about $x=1 / 2$ and has an increasing convex derivative $\left(q_{3}^{\prime}=-3 q_{4}\right)$ on $[1 / 2,1)$. Then, $A_{1}$ can be written

$$
\begin{aligned}
A_{1} & =\frac{1}{\pi z^{2}} \sum_{l=1}^{2 n-1} \phi_{2}\left(x_{l}\right)+\frac{3 \omega}{2 \pi^{2} z^{4}} \sum_{l=1}^{2 n-1} \phi_{4}\left(x_{l}\right)+2\left(\frac{n+\epsilon}{\pi z^{3}}\right) \sum_{l=1}^{2 n-1} q_{3}\left(x_{l}\right) \\
& =\Phi_{2}(n, \epsilon, \theta)+\Phi_{4}(n, \epsilon, \theta)+Q_{3}(n, \epsilon, \theta) .
\end{aligned}
$$

We discuss the cases $\epsilon>0, \epsilon<0$ separately.

(i) $\epsilon>0$ : Now $\Phi_{k}(n, \epsilon, \theta) \leq \Phi_{k}(n, 1 / 2,0)$. Indeed, $\phi_{2}, \phi_{4}$ are convex, increasing functions in $[0,1)$, and the $x_{l}$ are maximized for $\theta=0, \epsilon=1 / 2$. Similarly, the factors $z^{-k}$ are maximized for $\theta=0$. Finally, we have that for $k=2,4$,

$$
\Phi_{k}(n, \epsilon, \theta)=\frac{a_{k}}{[2(n+\theta)]^{k}} \sum_{l=1}^{2 n-1} \phi_{k}\left(x_{l}\right) \leq \Phi_{k}(n, 1 / 2,0)=\frac{a_{k}}{(2 n)^{k}} \sum_{l=1}^{2 n-1} \phi_{k}\left(\frac{l+1 / 2}{2 n}\right)
$$

with $a_{2}=1 / \pi, a_{4}=3 \omega /\left(2 \pi^{2}\right)$.

Also, using the properties of $q_{3}$, it follows that

$$
\begin{aligned}
& Q_{3}(n, \epsilon, \theta) \leq Q_{3}(n, 1 / 2,0) \\
& =2 \frac{(n+1 / 2)}{\pi(2 n)^{3}}\left(\sum_{l=1}^{n-1}\left[q_{3}\left(\frac{1}{2}+\frac{l-1 / 2}{2 n}\right)+q_{3}\left(\frac{1}{2}-\frac{l-1 / 2}{2 n}\right)\right]\right. \\
& \left.+q_{3}\left(\frac{1}{2}+\frac{n-1 / 2}{2 n}\right)\right) \\
& =\frac{n+1 / 2}{4 \pi n^{3}} q_{3}\left(1-\frac{1}{4 n}\right) .
\end{aligned}
$$

Combining results, we find that, for $\epsilon \geq 0$

$$
\begin{aligned}
\left|A_{1}\right| \leq & \frac{1}{4 \pi n^{2}} \sum_{l=1}^{2 n-1} \phi_{2}\left(\frac{l+1 / 2}{2 n}\right)+\frac{3 \omega}{32 \pi^{2} n^{4}} \sum_{l=1}^{2 n-1} \phi_{4}\left(\frac{l+1 / 2}{2 n}\right) \\
& +\frac{n+1 / 2}{4 \pi n^{3}} q_{3}\left(1-\frac{1}{4 n}\right) .
\end{aligned}
$$

(ii) $\epsilon<0$ : Now decrease $\theta$ to its minimum value, $\epsilon \leq \theta \leq 0$. This majorizes all terms:

$$
A_{1}(n, \epsilon, \theta) \leq A_{1}(n, \epsilon, \epsilon) .
$$


Next, observe that this is majorized for $\epsilon$ as small as possible, i.e. for $\epsilon=-1 / 2$. Indeed, now $x_{n}=1 / 2$ and

$$
\frac{d x_{l}}{d \epsilon}=\frac{n-l}{2(n+\epsilon)^{2}}
$$

is positive (negative) for $n>l(n<l)$. Since $\phi_{k}\left(x_{n+l}\right)>\phi_{k}\left(x_{n-l}\right)$, while $\frac{d x_{n+l}}{d \epsilon}=$ $-\frac{d x_{n-l}}{d \epsilon}$, we majorize $\phi_{k}\left(x_{n+l}\right)-\phi_{k}\left(x_{n-l}\right)$, and hence $\Phi_{k}$ by letting $\epsilon=-1 / 2$, its smallest possible value. On the other hand, the term $Q_{3}$ is now bounded above by $Q_{3}(n,-1 / 2,-1 / 2) \leq 0$, so we neglect it to get, for $\epsilon \leq 0$,

$$
\begin{aligned}
\left|A_{1}\right| & \leq \frac{1}{4 \pi(n-1 / 2)^{2}} \sum_{l=1}^{2 n-1} \phi_{2}\left(\frac{l-1 / 2}{2 n-1}\right) \\
& +\frac{3 \omega}{32 \pi^{2}(n-1 / 2)^{4}} \sum_{l=1}^{2 n-1} \phi_{4}\left(\frac{l-1 / 2}{2 n-1}\right) .
\end{aligned}
$$

Estimate for $A_{2}$. We let (with $a>0$ arbitrary)

$$
\begin{gathered}
f_{k}^{+}(x)=\sum_{p=0}^{\infty}(x+p)^{-k}, f_{1}^{+}(x)=\sum_{p=0}^{\infty}\left(\frac{1}{(x+p)}-\frac{1}{a+p}\right), \\
\phi_{k}^{+}=-\sum_{p=0}^{\infty} \frac{p}{(p+x)^{k}}=x f_{k}^{+}-f_{k-1}^{+}, \\
q_{k}^{+}=-\sum_{p=0}^{\infty} \frac{p(p+1)}{(p+x)^{k}}=(1-x) \phi_{k}^{+}+\phi_{k-1}^{+} .
\end{gathered}
$$

We have

$$
\begin{aligned}
A_{2} & =\frac{1}{\pi z^{2}}\left(f_{2}^{+}\left(y_{2}\right)+\phi_{2}^{+}\left(y_{1}\right)-\phi_{2}^{+}\left(y_{2}\right)\right) \quad\left(=\alpha_{2}\right) \\
& +\frac{3 \omega}{2 \pi^{2} z^{4}}\left(f_{4}^{+}\left(y_{2}\right)+\phi_{4}^{+}\left(y_{1}\right)-\phi_{4}^{+}\left(y_{2}\right)\right) \quad\left(=\alpha_{4}\right) \\
& +2 \frac{(n+\epsilon)}{\pi z^{3}}\left(q_{3}^{+}\left(y_{1}\right)-q_{3}^{+}\left(y_{2}\right)\right) \quad\left(=\alpha_{3}\right) .
\end{aligned}
$$

We note that $f_{k}^{+}$is positive and decreasing while $\phi_{k}^{+}, q_{k}^{+}$are negative and increasing for $x>0$. Again, we consider the cases $\epsilon>0$ and $\epsilon<0$ separately.

(i) For $\epsilon>0$, we have $y_{1}>y_{2}$. Then, for $\alpha_{2}, \alpha_{4}$ we must maximize $y_{1}$ and minimize $y_{2}$. This happens for $\epsilon=1 / 2, \theta=0$ (i.e. $y_{1}=1+1 / 4 n, y_{2}=1-1 / 4 n$ ), so

$$
\alpha_{2}+\alpha_{4} \leq \frac{1}{\pi(2 n)^{2}}\left(f_{2}^{+}\left(y_{1}\right)+\phi_{2}\left(\frac{1}{4 n}\right)\right)+\frac{3 \omega}{2 \pi^{2}(2 n)^{4}}\left(f_{4}^{+}\left(y_{1}\right)+\phi_{4}\left(\frac{1}{4 n}\right)\right) .
$$


For $\alpha_{3}$ we get, similarly,

$$
\begin{aligned}
\alpha_{3}(n, \epsilon, \theta) \leq \alpha_{3}(n, 1 / 2,0) \\
\quad=2 \frac{(n+1 / 2)}{\pi(2 n)^{3}}\left(\phi_{2}^{+}\left(y_{1}\right)-\phi_{2}^{+}\left(y_{2}\right)-\frac{1}{4 n}\left(\phi_{3}^{+}\left(y_{1}\right)+\phi_{3}^{+}\left(y_{2}\right)\right)\right) \\
\quad=2 \frac{(n+1 / 2)}{\pi(2 n)^{3}}\left(f_{2}^{+}\left(y_{1}\right)-f_{2}^{+}\left(y_{2}\right)+\phi_{2}\left(\frac{1}{4 n}\right)-\frac{1}{4 n}\left(\phi_{3}^{+}\left(y_{1}\right)+\phi_{3}^{+}\left(y_{2}\right)\right)\right) \\
\quad=2 \frac{(n+1 / 2)}{\pi(2 n)^{3}}\left(f_{2}^{+}\left(y_{1}\right)-f_{2}^{+}\left(y_{2}\right)+\phi_{2}\left(\frac{1}{4 n}\right)-\frac{2}{4 n} \phi_{3}^{+}\left(y_{2}\right)\right) \\
\quad=2 \frac{(n+1 / 2)}{\pi(2 n)^{3}}\left(f_{2}^{+}\left(y_{1}\right)-\left(1-\frac{1}{2 n}\right) f_{2}^{+}\left(y_{2}\right)+\phi_{2}\left(\frac{1}{4 n}\right)-\frac{1}{2 n}\left(1-\frac{1}{4 n}\right) f_{3}^{+}\left(y_{2}\right)\right)
\end{aligned}
$$

(ii) For $\epsilon<0, A_{2}$ is maximized at $\epsilon=\theta=-1 / 2$. Then

$$
\begin{aligned}
\alpha_{2}+\alpha_{4} \leq & \frac{1}{\pi(2 n-1)^{2}} f_{2}^{+}\left(1+\frac{1}{2(2 n-1)}\right) \\
& +\frac{3 \omega}{2 \pi^{2}(2 n-1)^{4}} f_{4}^{+}\left(1+\frac{1}{2(2 n-1)}\right) .
\end{aligned}
$$

Now, $\alpha_{3} \leq 0$, so it is neglected.

It is easy to show that the error term $\left|A_{1}+A_{2}\right|$ is maximal for $n=1$. Using the integral test, it can be easily shown that this term is a decreasing function of $n$ for $n$ sufficiently large (say $n>100$ ). The details, although lengthy, are elementary and they are ommitted. Numerical evaluation of the sums for $1<n \leq 100$ completes the proof. Thus we find that

$$
\left|A_{1}+A_{2}\right| \leq .958921+1.235903 \omega \leq 2.20 \text {. }
$$

This, used in (59), results in the bounds claimed in our theorem.

\section{REFERENCES}

1. T.M. Apostol, Introduction to Analytic Number Theory, 2nd printing, Springer, NY (1984). MR 55:7892 (1st printing)

2. M.V. Berry and J. Goldberg, Renormalization of curlicues, Nonlinearity $\underline{1}$, (1988), 1-26. MR 89b:58105

3. M.V. Berry, Random renormalization in the semiclassical long time limit of a precessing spin, Physica D $\underline{33}$ (1988), 26-33. MR 90c:81063

4. J.L. Callot and M. Diener, "Variations en spirale", Document du travail 6, p. 16-50, Oran, 1984.

5. K. Chandrasekharan, Elliptic Functions, Grundlehren der Math. Wissenschaften, 281, Springer-Verlag, Berlin, 1985. MR 87e:11058

6. J.G. van der Corput, Über Summen die mit den Elliptischen $\theta$-Funktionen zusammenhägen, I, II, Math. Ann. 87, (1922), 66-77; 90, (1923), 1-18.

7. J.G. van der Corput, Beweis einer approximativen Funktionalgleichung, Mathematische Zeitschrift, 28 (1928) 238-300.

8. E. A. Coutsias and N. D. Kazarinoff, Disorder, renormalisability, theta functions and Cornu spirals, Physica D $\underline{26}$, (1987) 295-310. MR 88h:11056

9. T. M. Dekking and M. Mendes-France, Uniform distributions modulo one: a geometric viewpoint, J. für die reine und angewandte Math., $\underline{329}$ (1981), 143-153. MR 83b:10062

10. J. M. Deshouillers, Geometric aspect of Weyl sums, Elementary and Analytic Theory of Numbers, p. 75-82, PWN, Warsaw 1985. MR 88d:11069

11. G. H. Hardy and J. E. Littlewood, Some problems of Diophantine approximation, Acta Math. 37, (1914), 193-238.

12. P. Henrici, Applied and Computational Complex Analysis, Vol. 2, Wiley, New York (1977). MR 56:12235 
13. J. B. Keller and C. Knessl, Asymptotic evaluation of oscillatory sums, European J. Appl. Math. 4 (1993), 361-379. MR 95f:11081

14. N. M. Korobov, Exponential Sums and their Applications, Mathematics and its Applications (Soviet Series), 으, Kluwer Academic Publishers, Boston, (1992). MR 93a:11068

15. L. Kronecker, Summierung der Gaussschen Reihen $\sum_{h=0}^{h=n-1} e^{2 h^{2} \pi i / n}$, Journal für die reine und angewandte Mathematik, 105(1889), 267-268.

16. D. H. Lehmer, Incomplete Gauss Sums, Mathematica, 23 (1976), 125-135. MR 55:2797

17. J.H. Loxton, The graphs of exponential sums, Mathematika, $\underline{30}$ (1983), 153-163. MR 85h: 11045

18. M. Mendes-France, The Planck constant of a curve, in Fractal Geometry and Analysis, p. 325-366, Bélair and Dubuc, eds., Kluwer Academic, (1991). MR 92f:58002

19. H. L. Montgomery, Ten Lectures on the Interface between Analytic Number Theory and Harmonic Analysis, CBMS $\underline{84}$, American Mathematical Society, (1994). MR 96i:11002

20. R. R. Moore and A. J. van der Poorten, On the thermodynamics of curves and other curlicues, in Miniconference on Geometry and Physics (Canberra 1989), M. N. Barber and M. K. Murray, eds., Proceedings of the Center of Mathematical Analysis, Australian Nat. Univ., vol. 22 , (1989), p.82-109. MR 91d:11168

21. L.J. Mordell, The approximate functional formula for the theta function, J. London Mat. Soc. 1 (1926) 68-72.

22. I.M. Vinogradov, The Method of Trigonometric Sums in the Theory of Numbers, translated from the Russian, revised and annotated by K.F. Roth and A. Davenport (Interscience, London, 1954). MR 15:941b

23. J. R. Wilton, The approximate functional formula for the theta function, J. London Math. Soc. $\underline{2}$ (1926), 177-180.

Department of Mathematics and Statistics, University of New Mexico, Albuquerque, New Mexico 87131

E-mail address, E. A. Coutsias: vageli@math.unm.edu 